BULLETIN (New Series) OF THE

AMERICAN MATHEMATICAL SOCIETY

Volume 42 Number 4 Pages $461-500$

S 0273-0979(05)01078-5

Article electronically published on July 7, 2005

\title{
COMPLEX SYMPLECTIC SPACES AND BOUNDARY VALUE PROBLEMS
}

\author{
W. N. EVERITT AND L. MARKUS \\ Dedicated to \\ Professor Jaroslav Kurzweil \\ on the occasion of his 80-th birthday
}

\begin{abstract}
This paper presents a review and summary of recent research on the boundary value problems for linear ordinary and partial differential equations, with special attention to the investigations of the current authors emphasizing the applications of complex symplectic spaces.

In the first part of the previous century, Stone and von Neumann formulated the theory of self-adjoint extensions of symmetric linear operators on a Hilbert space; in this connection Stone developed the properties of self-adjoint differential operators generated by boundary value problems for linear ordinary differential equations. Later, in diverse papers, Glazman, Krein and Naimark introduced certain algebraic techniques for the treatment of appropriate generalized boundary conditions. During the past dozen years, in a number of monographs and memoirs, the current authors of this expository summary have developed an extensive algebraic structure, complex symplectic spaces, with applications to both ordinary and partial linear boundary value problems.

As a consequence of the use of complex symplectic spaces, the results offer new insights into the theory and use of indefinite inner product spaces, particularly Krein spaces, from an algebraic viewpoint. For instance, detailed information is obtained concerning the separation and coupling of the boundary conditions at the endpoints of the intervals for ordinary differential operators (see the Balanced Intersection Principle), and the introduction of the generalized boundary conditions over the region for some elliptic partial differential operators (see the Harmonic operator).
\end{abstract}

\section{Contents}

1. Introduction to fundamental concepts: organization of the exposition

2. Motivating examples

2.1. Sturm-Liouville boundary value problems

2.2. Laplace boundary value problems

2.3. Comparison of boundary value problems

3. General theory of complex symplectic spaces

4. Finite dimensional complex symplectic spaces

Received by the editors March 10, 2005.

2000 Mathematics Subject Classification. Primary 51A50, 46A03, 37K05; Secondary 46C20, $35 \mathrm{~J} 40,34 \mathrm{~B} 05$.

Key words and phrases. Complex symplectic space, indefinite inner product space, topological linear space, ordinary and partial linear boundary value problem.

(C)2005 American Mathematical Society Reverts to public domain 28 years from publication 
4.1. Theory of separation and coupling for Lagrangian spaces

4.2. Applications for ordinary symmetric boundary conditions

5. Conclusions: comparisons, problems, conjectures

References

\section{INTRODUCTION TO FUNDAMENTAL CONCEPTS: ORGANIZATION OF THE EXPOSITION}

A complex symplectic space $S$ is a complex vector space with a prescribed symplectic product

$$
[\cdot: \cdot]: \mathrm{S} \times \mathrm{S} \rightarrow \mathbb{C},
$$

where $\mathbb{C}$ denotes the complex field, with the properties given in the following definition:

Definition 1.1. A complex symplectic space $S$ is a complex vector space, together with a prescribed symplectic form $[\cdot: \cdot]$, namely a sesqui-linear (or conjugate bilinear) complex-valued function

$$
\mathrm{u}, \mathrm{v} \rightarrow[\mathrm{u}: \mathrm{v}] \text { for } \mathrm{S} \times \mathrm{S} \rightarrow \mathbb{C}
$$

satisfying the following axioms, for all $\mathrm{u}, \mathrm{v}, \mathrm{w} \in \mathrm{S}$ and all complex numbers $c_{1}, c_{2} \in$ $\mathbb{C}$,

(1) $\left[c_{1} \mathbf{u}+c_{2} \mathbf{v}: \mathbf{w}\right]=c_{1}[\mathbf{u}: \mathbf{w}]+c_{2}[\mathbf{v}: \mathbf{w}]$ (linearity property in first argument),

(2) $[\mathrm{u}: \mathrm{v}]=-\overline{[\mathrm{v}: \mathrm{u}]}$ (skew-Hermitian property),

(3) $[\mathrm{u}: \mathrm{S}]=0$ implies $\mathrm{u}=0$ (non-degeneracy property).

Remark 1.2. Properties 1 and 2 of Definition 1.1 imply

$$
\left[\mathrm{u}: c_{1} \mathrm{v}+c_{2} \mathrm{w}\right]=\overline{c_{1}}[\mathrm{u}: \mathrm{v}]+\overline{c_{2}}[\mathrm{u}: \mathrm{w}] .
$$

Complex symplectic spaces are non-trivial generalizations (not merely complexifications) of the classical real symplectic spaces of Lagrangian and Hamiltonian mechanics; see [1], 20] and 23]. However these complex symplectic spaces have a much wider scope and admit new kinds of applications. For instance, for each positive integer $n \in \mathbb{N}:=\{1,2,3, \ldots\}$ there exist complex symplectic spaces (more than one) with dimension $n$ as a vector space over $\mathbb{C}$, whereas real symplectic spaces cannot be odd dimensional, and there exists a unique (up to symplectic isomorphism) real symplectic space for each even dimension $m$ as a vector space over the real field $\mathbb{R}$.

The study of these complex symplectic spaces is motivated by the boundary value problems for linear ordinary and partial differential operators with complex coefficients and is closely related to the corresponding spectral analysis within complex Hilbert spaces; see the results of Everitt and Markus, especially [10] and [11, and [13].

The particular results which guided our approach to the introduction and development of complex symplectic spaces are to be seen in the work of Akhiezer and Glazman [2, Appendix 1], Coddington and Levinson [5, Chapters 7 to 11], Dunford and Schwartz [6, Chapters XII and XIII], Everitt and Markus 9, Naimark 27, Chapter V], and Titchmarsh [32] and [33]. 
For the analysis of ordinary differential expressions (i.e. formal differential operators), finite dimensional complex symplectic spaces are adequate, but for elliptic partial differential expressions, infinite dimensional symplectic spaces are required. In this exposition we endeavor to present completely and precisely all statements of definitions, theorems and other results, but usually omit all proofs (except for occasional sketches), and then refer to the recent extensive literature for further details and explanations.

Here we record some of the main results that have followed from this study of complex symplectic spaces:

(i) The generalized Glazman-Krein-Naimark (GKN) Theorem [see Section 1 . Theorem 1.14 and Remark 1.15, which extends the original theorem, as initially restricted to self-adjoint ordinary differential operators, to the determination of all self-adjoint extensions of closed symmetric operators in abstract Hilbert space; see [10, Section II, Theorem 1] and [14, Theorem 5.2].

(ii) The Balanced Intersection Principle [see Section 4.1], which resolves the problem of determining the canonical forms for all possible symmetric (formally self-adjoint) boundary conditions for general Lagrange symmetric ordinary differential expressions (covering both the regular and singular cases); see 10, Preface and Chapter V, Section 4].

(iii) The symplectic version of the generalized GKN Theorem [see Section 2.2, which explores and classifies all self-adjoint extensions of a symmetric operator in terms of the complete Lagrangians in the corresponding boundary complex symplectic space, in particular for symmetric operators generated by ordinary and partial differential expressions; see [10, Section II, Theorem 1] and [13, Section $3]$.

(iv) The Harmonic operator [see Section 2.2, especially (2.62) and (2.63)], which is an unusual example of a self-adjoint operator generated by an appropriate elliptic partial differential expression on bounded Euclidean regions, here illustrated by the Laplace regular partial differential expression on a planar disk. This Harmonic operator is not specified by data evaluated on the boundary circle, and moreover has a non-empty essential spectrum; see [13, Section 4, Definition $4.2]$ and [15.

$(v)$ The symplectic weak topology [see Section 3, and also Definition 1.12, which is intrinsically defined on each complex symplectic space $\mathbf{S}$ and defines $\mathrm{S}$ as a locally convex topological vector space (not necessarily metrizable); see [14, Section 3].

In the introductory Section 1 of this survey we first present the most basic concepts and definitions for complex symplectic spaces and their Lagrangian subspaces with the corresponding linear algebra (known as symplectic algebra). We illuminate this symplectic algebra by its applications to linear operator theory through a number of examples including the construction of symplectic spaces via Hilbert spaces. In particular, the boundary space of a symmetric operator in Hilbert space theory is important for asserting and describing the Glazman-Krein-Naimark (GKN)Theorem, as generalized by Zettl [35], and then by Everitt and Markus [10, Section III, Part 2] and [11, Appendix, Corollary 1].

In Section 2 we describe, in appropriate detail, the boundary value theory for two important motivating examples of second-order differential expressions - the 
general Sturm-Liouville ordinary differential operator and the Laplace partial differential operator. In particular, we interpret the associated boundary value problems through symplectic algebra. In addition, there is an indication of the application of these techniques to higher order ordinary differential expressions, and these ideas are carried further in Section 4.2 using the even more general notation of quasidifferential coefficients.

In Section 3 we develop more thoroughly the general theory of complex symplectic spaces of arbitrary dimension (finite or infinite). In particular, we define and compare various topologies, including the symplectic weak topology and the Hilbert topology. Also we investigate the interrelations among the symplectic invariants when they are infinite cardinal numbers; see 11], 12, 13] and 14.

In Section 4 we review some important properties of finite dimensional spaces and apply these methods to regular and singular boundary value problems for general Lagrange symmetric ordinary differential expressions. In particular we emphasize that each self-adjoint operator thus determined is necessarily defined through (generalized) symmetric boundary conditions, and we describe an explicit classification of such boundary conditions by their degree of coupling (or separation) at the interval endpoints.

Section 5 offers some conclusions about the topics, results, and problems summarized in this paper and then presents some new directions for research, including open problems and a few uncertain conjectures.

Notation 1.3. We use the normal fonts for text (roman) and mathematics (italic); for symplectic spaces we use sans serif font. In case a space has the properties of being both a Hilbert space and a symplectic space, we use sans serif font to represent both spaces; for examples see Definition 1.1 and Examples 1.9 and 1.13 .

To emphasize the analogy between a complex symplectic space $S$ and a Hermitian scalar product space [28, Chapter 12, Section 12.1], we often refer to the symplectic product $[\mathrm{u}: \mathrm{v}]$ with symplectic orthogonality in case $[\mathrm{u}: \mathrm{v}]=0$. Hence the nondegeneracy condition of item 3 in Definition 1.1 asserts that only the zero vector of $\mathrm{S}$ is symplectically orthogonal to every vector in S.

In the same spirit, a conjugate bilinear form on a complex vector space $\mathrm{S}$, satisfying just conditions 1 and 2 of Definition 1.1, is called a "degenerate symplectic product", although this defines $S$ as a complex symplectic space only if non-degeneracy condition 3 of Definition 1.1 also holds.

Remark 1.4. The usual concepts and constructions for vector spaces apply, with some exceptions, to complex symplectic spaces $S$ with symplectic product $[\cdot: \cdot]$; see [11, Section 1] and [14, Section 1].

For instance, two complex symplectic spaces $S$ with symplectic product [ : : · and $S_{1}$ with $[\cdot: \cdot]_{1}$ are symplectically isomorphic in case there exists a (complex) linear bijective map $F$ of $S$ onto $S_{1}$ which preserves symplectic products; that is

$$
[\mathrm{u}: \mathrm{v}]=[F \mathrm{u}: F \mathrm{v}]_{1} \text { for all } \mathrm{u}, \mathrm{v} \in \mathrm{S} .
$$

If $\mathrm{S}$ and $\mathrm{S}_{1}$ are the same complex symplectic space, then $F$ is a symplectic automorphism of S, and we can consider the group of all symplectic automorphisms of S; see [25].

As another instance, let $\mathrm{V} \subset \mathrm{S}$ be a linear submanifold of $\mathrm{S}$; that is

$$
\mathrm{V}=\operatorname{span}\{\mathrm{V}\}
$$


(for finite complex linear combinations of elements of $\mathrm{V}$ ). Then $\mathrm{V}$ is a symplectic subspace of $\mathrm{S}$ just in case the inherited (or induced) symplectic product on $\mathrm{V}$ is non-degenerate.

Definition 1.5. Let $\mathrm{V}_{1}$ and $\mathrm{V}_{2}$ be linear submanifolds of the complex symplectic space $\mathrm{S}$ with $[\cdot: \cdot]$. Assume that

$$
\mathrm{S}=\operatorname{span}\left\{\mathrm{V}_{1}, \mathrm{~V}_{2}\right\} \text { and } \mathrm{V}_{1} \cap \mathrm{V}_{2}=0 ;
$$

that is, $\mathrm{S}$ is the direct sum of $\mathrm{V}_{1}$ and $\mathrm{V}_{2}$.

If further $V_{1}$ and $V_{2}$ are symplectically orthogonal,

$$
\left[\mathrm{V}_{1}: \mathrm{V}_{2}\right]=0
$$

then we write

$$
\mathrm{S}=\mathrm{V}_{1} \oplus \mathrm{V}_{2}
$$

and note that, in this case, both $\mathrm{V}_{1}$ and $\mathrm{V}_{2}$ are symplectic subspaces of $\mathrm{S}$.

Definition 1.6. For each (non-empty) subset $\vee \subset S$, the symplectic orthocomplement of $\mathrm{V}$ is defined by

$$
\mathrm{V}^{\#}:=\{\mathrm{u} \in \mathrm{S}:[\mathrm{u}: \mathrm{V}]=0\},
$$

and we note that $\mathrm{V} \#$ is a linear submanifold of $\mathrm{S}$.

Note that if $\mathrm{S}=\mathrm{V}_{1} \oplus \mathrm{V}_{2}$ as in (1.7), then

$$
\mathrm{V}_{1}^{\#}=\mathrm{V}_{2} \text { and } \mathrm{V}_{2}^{\#}=\mathrm{V}_{1} \text {. }
$$

Definition 1.7. Let $S$ with $[\cdot: \cdot]$ be a complex symplectic space. Then a linear manifold $\mathrm{L} \subset \mathrm{S}$ is called Lagrangian in case

$$
[\mathrm{L}: \mathrm{L}]=0 \text { (also written } \mathrm{L} \subset \mathrm{L}^{\#} \text { ). }
$$

Further a Lagrangian manifold $L$ is called complete in case

$$
\mathrm{u} \in \mathrm{S} \text { and }[\mathrm{u}: \mathrm{L}]=0 \text { imply } \mathrm{u} \in \mathrm{L} \text { (also written } \mathrm{L}=\mathrm{L}^{\#} \text { ). }
$$

We next present several examples for the construction of complex symplectic spaces, leading to the important GKN-Theorem, as generalized by Everitt and Markus; see [9], 10, Section III, Part 2] and [11, Appendix, Corollary 1] for additional details of these examples.

Example 1.8. Consider the complex vector space $\mathbb{C}^{n}$, consisting of all complex (row) $n$-tuples, so $\operatorname{dim}\left(\mathbb{C}^{n}\right)=n \in \mathbb{N}$, as usual. Let $K$ be a given complex $n \times n$ matrix which satisfies the conditions:

$$
\begin{cases}K=-K^{*} & \text { (where } K^{*} \text { is the conjugate transpose) } \\ \operatorname{det}(K) \neq 0 & \text { (non-singular). }\end{cases}
$$

Define the symplectic product of vectors $\mathrm{u}, \mathrm{v} \in \mathbb{C}^{n}$ by

$$
[\mathrm{u}: \mathrm{v}]:=\mathrm{u} K \mathrm{v}^{*} \text {. }
$$

Then, from Definition 1.1] $\mathbb{C}^{n}$ with [.: ·] is a complex symplectic space, which we here denote by $\mathrm{S}$. 
Since $(i K)=(i K)^{*}$ is a Hermitian matrix, it is known, by familiar linear algebra (see [4, Chapter VIII]) that there exists an appropriate basis for $\mathbb{C}^{n}$, in terms of which we can assume, without loss of generality,

$$
i K=\left[\begin{array}{cc}
I_{q} & 0 \\
0 & -I_{p}
\end{array}\right] \text { or } K=\left[\begin{array}{cc}
-i I_{q} & 0 \\
0 & i I_{p}
\end{array}\right]
$$

for some non-negative integers $p$ and $q$. (Here $I_{p}$ denotes the identity matrix of size $p \geq 0$, which is omitted if $p=0$, and similarly for $I_{q}$.)

In terms of these co-ordinates in $\mathrm{S}$, we can define a symplectic orthogonal decomposition of $\mathrm{S}$ into two symplectic subspaces $\mathrm{H}_{ \pm}$,

$$
\mathrm{S}=\mathrm{H}_{-} \oplus \mathrm{H}_{+} \text {. }
$$

In more detail, each vector, say

$$
\mathrm{u}=\left(\mathrm{u}_{1}, \ldots, \mathrm{u}_{q}, \mathrm{u}_{q+1}, \ldots, \mathrm{u}_{n}\right) \in \mathrm{S},
$$

has a unique decomposition

$$
\mathrm{u}=\mathrm{u}_{-}+\mathrm{u}_{+}
$$

where

$$
\mathrm{u}_{-}=\left(\mathrm{u}_{1}, \ldots, \mathrm{u}_{q}, 0, \ldots, 0\right) \text { and } \mathrm{u}_{+}=\left(0, \ldots, 0, \mathrm{u}_{q+1}, \ldots, \mathrm{u}_{n}\right) .
$$

Then define

$$
\mathrm{H}_{-}:=\left\{\mathrm{u} \in \mathrm{S}: \mathrm{u}_{+}=0\right\} \text { and } \mathrm{H}_{+}:=\left\{\mathrm{u} \in \mathrm{S}: \mathrm{u}_{-}=0\right\} .
$$

Next calculate

$$
[\mathrm{u}: \mathrm{v}]=\left[\mathrm{u}_{-}+\mathrm{u}_{+}: \mathrm{v}_{-}+\mathrm{v}_{+}\right]=\left[\mathrm{u}_{-}: \mathrm{v}_{-}\right]+\left[\mathrm{u}_{+}: \mathrm{v}_{+}\right]=-i \mathrm{u}_{-} \mathrm{v}_{-}^{*}+i \mathrm{u}_{+} \mathrm{v}_{+}^{*} \text {. }
$$

Accordingly, we can define scalar products $\langle\cdot, \cdot\rangle_{ \pm}$in $\mathrm{H}_{ \pm}$, respectively:

$$
\left\langle\mathrm{u}_{-}, \mathrm{v}_{-}\right\rangle_{-}:=i\left[\mathrm{u}_{-}: \mathrm{v}_{-}\right] \text {and }\left\langle\mathrm{u}_{+}, \mathrm{v}_{+}\right\rangle_{+}:=-i\left[\mathrm{u}_{+}: \mathrm{v}_{+}\right] .
$$

In this way $\mathrm{H}_{ \pm}$are each a Hilbert space.

While the particular Hilbert spaces $\mathrm{H}_{ \pm}$are not uniquely specified by (1.15), the non-negative integers

$$
\begin{aligned}
& \mathbf{p}:=\operatorname{dim}\left(\mathrm{H}_{+}\right) \\
& \mathbf{q}:=\operatorname{dim}\left(\mathrm{H}_{-}\right)
\end{aligned}
$$

are uniquely determined by $\mathrm{S}$ (see discussion following Example 1.9) and are known as the positivity and negativity indexes of $\mathbf{S}$. That is, $\mathbf{p}$ and $\mathbf{q}$ are symplectic invariants of $\mathbf{S}$; in fact the pair $(\mathbf{p}, \mathbf{q})$ constitute a complete set of invariants which characterize $\mathbf{S}$ up to symplectic isomorphism. Other convenient symplectic invariants of $\mathrm{S}$ are

$$
\begin{gathered}
\operatorname{dim}(\mathrm{S})=\mathbf{p}+\mathbf{q}, \text { Excess or } \operatorname{Ex}(\mathrm{S}):=\mathbf{p}-\mathbf{q} \\
\Delta(\mathrm{S}):=\min \{\mathbf{p}, \mathbf{q}\}, \text { the Lagrangian index of } \mathrm{S} .
\end{gathered}
$$

From another direction, start with any $n$-dimensional complex symplectic space $\mathrm{S}_{1}$ with symplectic product $[\cdot: \cdot]_{1}$. Then there exists a linear isomorphism of the vector space $S_{1}$ onto $\mathbb{C}^{n}$, and this map induces a symplectic product [.: · $]$ on $\mathbb{C}^{n}$ - necessarily specified by some non-singular skew-Hermitian matrix, say $K_{1}$, satisfying conditions (1.12). Therefore $S_{1}$ with $[\cdot: \cdot]_{1}$ is symplectic isomorphic to $\mathbb{C}^{n}$ with $[\cdot: \cdot]$, as described above. 
The analysis of Example 1.8 can be reversed to construct a complex symplectic space as the direct sum of two Hilbert spaces.

Example 1.9. Let $\mathrm{H}_{-}$and $\mathrm{H}_{+}$be complex Hilbert spaces (finite or infinite dimensional), with corresponding scalar products and norms $\langle\cdot, \cdot\rangle_{ \pm}$and $\|\cdot\|_{ \pm}$, respectively, and consider the direct sum Hilbert space

$$
\mathrm{S}:=\mathrm{H}_{-} \oplus \mathrm{H}_{+},
$$

so that each vector $\mathrm{u} \in \mathrm{S}$ has a unique representation as an ordered couple

$$
\mathrm{u}=\left(\mathrm{u}_{-}, \mathrm{u}_{+}\right) \text {, with } \mathrm{u}_{-} \in \mathrm{H}_{-} \text {and } \mathrm{u}_{+} \in \mathrm{H}_{+},
$$

and the vectors $\left(\mathbf{u}_{-}, 0\right)$ and $\left(0, \mathbf{u}_{+}\right)$are orthogonal in $\mathbf{S}$.

Then $\mathrm{S}$ is a Hilbert space with norm given by

$$
\|\mathrm{u}\|_{\mathrm{S}}^{2}=\left\|\mathrm{u}_{-}\right\|_{-}^{2}+\left\|\mathrm{u}_{+}\right\|_{+}^{2},
$$

and furthermore $S$ is a complex symplectic space with symplectic product of $u=$ $\left(\mathrm{u}_{-}, \mathrm{u}_{+}\right)$and $\mathrm{v}=\left(\mathrm{v}_{-}, \mathrm{v}_{+}\right)$:

$$
[\mathrm{u}: \mathrm{v}]=-i\left\langle\mathrm{u}_{-}, \mathrm{v}_{-}\right\rangle_{-}+i\left\langle\mathrm{u}_{+}, \mathrm{v}_{+}\right\rangle_{+} .
$$

Thus for $\mathrm{u}_{ \pm}, \mathrm{v}_{ \pm} \in \mathrm{H}_{ \pm}$, both respectively, compare (1.19) and (1.20):

$$
\left[\left(\mathrm{u}_{-}, \mathrm{u}_{+}\right):\left(\mathrm{v}_{-}, \mathrm{v}_{+}\right)\right]=-i\left\langle\mathrm{u}_{-}, \mathrm{v}_{-}\right\rangle_{-}+i\left\langle\mathrm{u}_{+}, \mathrm{v}_{+}\right\rangle_{+} .
$$

In this sense each of $\mathrm{H}_{-}$and $\mathrm{H}_{+}$is a Hilbert subspace of $\mathrm{S}$ and also a symplectic subspace of S. Moreover (1.25) yields an orthogonal direct sum of $\mathrm{H}_{-}$and $\mathrm{H}_{+}$, with regard to both the Hilbert scalar product and the symplectic product. We summarize this situation by asserting that $\mathrm{S}$ has a Hilbert structure, as designated by the Hilbert pair $\left\{\mathrm{H}_{-}, \mathrm{H}_{+}\right\}$- non-unique since other Hilbert pairs can define the same complex symplectic space $\mathrm{S}$.

Remark 1.10. According to Example 1.8, each finite dimensional complex symplectic space has a Hilbert structure, noting (1.15). However, there exist infinite dimensional complex symplectic spaces which do not possess any Hilbert structure; see [3, Chapter I, Example 11.3] and [14, page 39].

In case the complex symplectic space $\mathrm{S}$ does have a Hilbert structure, say as in (1.15) or (1.25),

$$
\mathrm{S}=\mathrm{H}_{-} \oplus \mathrm{H}_{+},
$$

the positivity and negativity indexes, defined respectively by

$$
\mathbf{p}:=\operatorname{dim}\left(\mathbf{H}_{+}\right) \text {and } \mathbf{q}:=\operatorname{dim}\left(\mathbf{H}_{-}\right) \text {, }
$$

are known to be independent of the choice of the Hilbert pair $\left\{\mathrm{H}_{-}, \mathrm{H}_{+}\right\}$decomposing S.

However, for every complex symplectic space S, without reference to any Hilbert structure, we can also give an intrinsic definition of these two symplectic invariants $\mathbf{p}$ and $\mathbf{q}$. First note that for each vector $\mathbf{u} \in \mathrm{S}$, the symplectic product $[\mathbf{u}: \mathbf{u}]$ is purely imaginary and so can be classified by its imaginary part:

$$
\begin{cases}\operatorname{Im}([u: u])>0 & u \text { is a positive vector } \\ \operatorname{Im}([u: u])<0 & \mathbf{u} \text { is a negative vector } \\ \operatorname{Im}([\mathbf{u}: \mathbf{u}])=0 & \mathbf{u} \text { is a neutral vector. }\end{cases}
$$


Then a linear manifold $\vee \subset S$ is called a positivity space in case (compare (1.29)): (1.33)

$\left\{\begin{array}{l}\operatorname{Im}([\mathrm{v}: \mathrm{v}])>0 \text { for all non-zero } \mathrm{v} \in \mathrm{V} \text {, and } \\ \|\mathrm{v}\|_{\mathrm{V}}^{2}=-i[\mathrm{v}: \mathrm{v}] \text { specifies a norm for which } \mathrm{V} \text { is a (complete) Hilbert space. }\end{array}\right.$

Similarly, a linear manifold $\mathrm{W} \subset \mathrm{S}$ is called a negativity space in case:

$\{\operatorname{Im}([\mathrm{w}: \mathrm{w}])<0$ for all non-zero $w \in W$, and

$\left\{\|\mathrm{w}\|_{\mathrm{W}}^{2}=i[\mathrm{w}: \mathrm{w}]\right.$ specifies a norm for which $\mathrm{W}$ is a (complete) Hilbert space.

Definition 1.11. Let $S$ be a complex symplectic space, with symplectic product $[\cdot: \cdot]$. Then the positivity index $\mathbf{p}$ and the negativity index $\mathbf{q}$ of $\mathbf{S}$ are the cardinal numbers:

$$
\begin{array}{r}
\mathbf{p}:=\sup \{\operatorname{dim}(\mathrm{P}): \mathrm{P} \text { is a (closed) positivity space in } \mathrm{S}\} \\
\mathbf{q}:=\sup \{\operatorname{dim}(\mathrm{N}): \mathrm{N} \text { is a (closed) negativity space in } \mathrm{S}\} .
\end{array}
$$

Here the terminology "closed space" refers to the symplectic weak topology on $\mathrm{S}$ (as defined next - and which coincides with a closed Hilbert subspace in the case when S has a Hilbert structure; see Section 3).

Regardless of the existence of a Hilbert structure or not, every complex symplectic space $\mathbf{S}$ admits a distinguished intrinsic topology (not necessarily metrizable), and this defines $S$ as a locally convex topological vector space [14, Section 3, Theorem 3.1], namely the symplectic weak topology as specified next; see [6] and 22.

Definition 1.12. Let $S$ with $[\cdot: \cdot]$ be a complex symplectic space. Consider for each vector $w \in S$ the complex linear functional

$$
F_{\mathrm{w}}: \mathrm{u} \rightarrow[\mathrm{u}: \mathrm{w}] \text { to give } \mathrm{S} \rightarrow \mathbb{C} .
$$

Then the symplectic weak topology on $S$ is the (unique) weakest topology for which every such linear functional $\left\{F_{\mathrm{w}}: \mathrm{w} \in \mathrm{S}\right\}$ is continuous.

Of course, the symplectic weak topology coincides with the Hilbert norm topology on $\mathrm{S}$ when $\mathrm{S}$ is finite dimensional (a finite dimensional vector space admits a unique topology as a topological vector space; see [28, Chapter 1, Section 1.19]). However, if $\mathrm{S}=\mathrm{H}_{-} \oplus \mathrm{H}_{+}$is infinite dimensional, with a Hilbert structure, then the symplectic weak topology is different from the Hilbert topology (i.e. strictly weaker); see discussions in Section 3 .

The next example explains the relation between the Stone - von Neumann theory of symmetric operators on an abstract complex Hilbert space $H$ and the theory of complex symplectic spaces and their symplectic algebra. In particular, for each specified symmetric operator $T_{0}$, with dense domain $D\left(T_{0}\right) \subset H$, we define the boundary complex symplectic space $\mathrm{S}$ for $T_{0}$.

Example 1.13. Let $H$ be a complex Hilbert space (of arbitrary dimension), with scalar product and norm denoted by $\langle f, g\rangle$ and $\|f\|=|\langle f, f\rangle|^{1 / 2}$ for all vectors $f, g \in H$. Also let $T_{0}$ be a closed symmetric operator with dense domain $D\left(T_{0}\right) \subset H$ (if a symmetric operator with a dense domain on $H$ is not closed, replace it by its closure and proceed without loss of generality). Next let $T_{1}$ on domain $D\left(T_{1}\right) \subset H$ be the adjoint of $T_{0}$ in $H$, as indicated by

$$
T_{1}=T_{0}^{*},
$$


and then $T_{0}$ is the restriction of $T_{1}$ to $D\left(T_{0}\right) \subset D\left(T_{1}\right)$. Furthermore, in this situation (see [6, [10, Section II] and [11, Appendix]) it follows that

$$
T_{0}=T_{1}^{*} \text {. }
$$

Moreover, every self-adjoint operator $T$ on $D(T)$, which is an extension of $T_{0}$ on $D\left(T_{0}\right)$, and thence a restriction of $T_{1}$ on $D\left(T_{1}\right)$, satisfies the bounds, respectively:

$$
T_{0} \subseteq T=T^{*} \subseteq T_{1} \text { on } D\left(T_{0}\right) \subseteq D(T)=D\left(T^{*}\right) \subseteq D\left(T_{1}\right) .
$$

Accordingly we call $T_{0}$ and $T_{1}$ the minimal and maximal operators for $T_{0}$.

The linear manifold $D\left(T_{1}\right)$ is not a closed subspace of the Hilbert space $H$, but it is nevertheless a Hilbert space relative to the $T_{1}$-graph norm with the following scalar product:

$$
\langle f, g\rangle_{D}:=\langle f, g\rangle+\left\langle T_{1} f, T_{1} g\right\rangle \text { for all } f, g \in D\left(T_{1}\right),
$$

with the norm defined by

$$
\|f\|_{D}^{2}:=\langle f, f\rangle_{D} .
$$

Further $D\left(T_{1}\right)$ bears a degenerate symplectic product

$$
[f, g]_{D}:=\left\langle T_{1} f, g\right\rangle-\left\langle f, T_{1} g\right\rangle \text { for all } f, g \in D\left(T_{1}\right),
$$

where we note that, since $T_{0}$ is symmetric on $D\left(T_{0}\right) \subseteq D\left(T_{1}\right)$,

$$
[f, g]_{D}=0 \text { for all } f, g \in D\left(T_{0}\right) .
$$

The main fact in this theory is the Stone-von Neumann direct sum decomposition within the Hilbert space $D\left(T_{1}\right)$ :

$$
D\left(T_{1}\right)=D\left(T_{0}\right) \oplus N^{-} \oplus N^{+} .
$$

Here we define the deficiency spaces $N^{ \pm}$of $T_{0}$ by

$$
N^{ \pm}:=\left\{f \in D\left(T_{1}\right): T_{1} f= \pm i f\right\},
$$

which are closed subspaces of $D\left(T_{1}\right)$ (and also of $H$ ); from these deficiency spaces we further define the deficiency indices $d^{ \pm}$of $T_{0}$, as cardinal numbers, respectively by

$$
d^{ \pm}:=\operatorname{dim}\left(N^{ \pm}\right) .
$$

It is known that there are self-adjoint extensions $T$ of $T_{0}$, equivalently self-adjoint restrictions of $T_{1}$, if and only if

$$
d^{-}=d^{+}
$$

in this case, see (1.40). The symmetric operator $T_{0}$ is self-adjoint if and only if

$$
d^{-}=d^{+}=0 \text { and then } T_{0}^{*}=T_{1}=T_{0} .
$$

From our viewpoint the most important aspect of the direct sum decomposition (1.45) of $D\left(T_{1}\right)$ is that the summands are mutually orthogonal linear manifolds within the Hilbert space $D\left(T_{1}\right)$, both with respect to the Hilbert scalar product $\langle\cdot, \cdot\rangle_{D}$ and to the symplectic product $[\cdot: \cdot]_{D}$. It follows that

$$
D\left(T_{0}\right)=\left\{f \in D\left(T_{1}\right):\left[f: D\left(T_{1}\right)\right]_{D}=0\right\} .
$$

This conclusion (1.50) makes it possible to define the complex symplectic space $S$ as the quotient or identification space

$$
\mathrm{S}:=D\left(T_{1}\right) / D\left(T_{0}\right),
$$


whose elements are cosets $f=\left\{f+D\left(T_{0}\right)\right\}, g=\left\{g+D\left(T_{0}\right)\right\}$, etc. That is, the natural projection $\Psi$ carries $D\left(T_{1}\right)$ onto $\mathrm{S}$, according to the definition:

$$
\Psi: D\left(T_{1}\right) \rightarrow D\left(T_{1}\right) / D\left(T_{0}\right) \text { as defined by } f \rightarrow \Psi f:=\mathrm{f}=\left\{f+D\left(T_{0}\right)\right\} .
$$

Using representative functions $f, g \in D\left(T_{1}\right)$ define the non-degenerate symplectic product on $\mathrm{S}$ by

$$
[\mathrm{f}: \mathrm{g}]:=\left[f+D\left(T_{0}\right), g+D\left(T_{0}\right)\right]_{D}=[f, g]_{D}
$$

(independent of the choice of functions $f \in \mathrm{f}$ and $g \in \mathrm{g}$ ).

There is an important connection between the deficiency indices $d^{ \pm}$of the minimal operator $T_{0}$ and the positivity index $\mathbf{p}$ (see (1.35) and the negativity index $\mathbf{q}$ (see (1.36) ) of $\mathrm{S}$ as defined by (1.51) (note $\mathrm{S}$ has a Hilbert structure specified by the Hilbert pair $\left.\left\{N^{-}, N^{+}\right\}\right)$:

$$
\mathbf{p}=d^{+} \text {and } \mathbf{q}=d^{-} \text {. }
$$

We name the complex symplectic space $S$ with $[\cdot: \cdot]$ to be the boundary space of $T_{0}$. With this terminology we can now state the abstract version of the GlazmanKrein-Naimark (GKN) Theorem (see [27, Chapter V, Section 17 and 18]), as generalised by Everitt and Markus; see [10, Section III, Part 2] and [11, Appendix].

Theorem 1.14. Let $T_{0}$ be a closed symmetric operator with dense domain $D\left(T_{0}\right)$ in a complex Hilbert space $H$, as above.

Consider the $H$-adjoints

$$
\begin{gathered}
T_{1}=T_{0}^{*} \text { on } D\left(T_{1}\right) \subset H \\
T_{0}=T_{1}^{*} \text { on } D\left(T_{0}\right) \subseteq D\left(T_{1}\right) .
\end{gathered}
$$

Further consider the boundary complex symplectic space of $T_{0}$

$$
\mathrm{S}=D\left(T_{1}\right) / D\left(T_{0}\right)
$$

with symplectic product $[\cdot: \cdot]$ as defined in (1.51) and (1.53).

Then there exists a natural one-to-one correspondence between the set $\{T\}$ of all self-adjoint extensions of $T_{0}$ and the set $\{\mathrm{L}\}$ of all complete Lagrangians $\mathrm{L} \subset \mathrm{S}$. Namely, for each self-adjoint operator $T$ on $D(T)$ (necessarily a restriction of $T_{1}$ on $\left.D\left(T_{1}\right)\right)$, define the corresponding complete Lagrangian $\mathrm{L} \subset \mathrm{S}$ by

$$
\mathrm{L}:=\{\mathrm{f} \in \mathrm{S}: f \in D(T)\} \text { (also written } \mathrm{L}=\Psi D(T) \text { as in (1.52) })
$$

and then

$$
D(T)=\left\{f \in D\left(T_{1}\right): \mathrm{f}=\left\{f+D\left(T_{0}\right)\right\} \in \mathrm{L}\right\} .
$$

Remark 1.15. Note that $\mathrm{L}$ is the image of $D(T)$ under the natural projection $\Psi$ of $D\left(T_{1}\right)$ onto $\mathrm{S}$, and, as in (1.52),$D(T)$ is the inverse image of $\mathrm{L}$ for this natural projection.

Consequently the set $\{T\}$ is empty if and only if the set $\{L\}$ is empty, and it is known that this circumstance holds if and only if the positivity and negativity indices (see (1.31), (1.35), (1.36) and (1.47) ) are unequal, i.e.

$$
\mathbf{q} \neq \mathbf{p} \text { i.e. } d^{-} \neq d^{+} \text {. }
$$


Equivalently, the set $\{T\}$ is not empty if and only if the set $\{L\}$ is not empty, and this circumstance holds if and only if the deficiency indices $d^{ \pm}$of $T_{0}$ (see (1.47), are equal), i.e.

$$
d^{-}=d^{+} \text {i.e. } \mathbf{q}=\mathbf{p}
$$

\section{Motivating EXAmples}

2.1. Sturm-Liouville boundary value problems. As indicated in Section 1 we describe here the construction of Sturm-Liouville ordinary differential operators, which leads to the definition of important finite dimensional complex symplectic spaces with Hilbert structure. This account is based on the general theory of Lagrange symmetric quasi-differential expressions (see [27, Chapter V], 17, , 9] and 24]) and covers both the regular and singular cases of Sturm-Liouville differential expressions. We follow a modern path (see [9]) for the analysis of the boundary value theory for ordinary differential expressions and then introduce the symplectic algebraic interpretation in the latter part of this section.

Suppose we are given the open interval $(a, b)$, bounded or unbounded, of the real line $\mathbb{R}$ and the three coefficients $p, q, w$ satisfying the properties

$$
\begin{cases}(i) & p, q, w:(a, b) \rightarrow \mathbb{R} \\ (\text { ii }) & p^{-1}, q, w \in L_{\text {loc }}^{1}(a, b) \\ (\text { iii }) & w(x)>0 \text { for almost all } x \in(a, b) .\end{cases}
$$

These coefficients determine the Sturm-Liouville differential equation

$$
-\left(p(x) y^{\prime}(x)\right)^{\prime}+q(x) y(x)=\lambda w(x) y(x) \text { for almost all } x \in(a, b),
$$

where $\lambda \in \mathbb{C}$ is a complex-valued spectral parameter and $w$ is the weight function.

Given the interval $(a, b)$ and the set of Sturm-Liouville coefficients $\{p, q, w\}$, one can define the associated quasi-differential expression $M[\cdot]$ on the domain

$$
D(M):=\left\{f:(a, b) \rightarrow \mathbb{C}: f, p f^{\prime} \in A C_{\mathrm{loc}}(a, b)\right\}
$$

and

$$
M[f](x) \equiv-\left(p(x) f^{\prime}(x)\right)^{\prime}+q(x) f(x) \text { for all } x \in(a, b) \text { and } f \in D(M) .
$$

The above minimal conditions on the set of coefficients $\{p, q, w\}$ imply that the Sturm-Liouville differential equation has a solution to any initial value problem at a point $c \in(a, b)$ (see the existence theorem in [27, Chapter V, Section 15]); i.e. given two complex numbers $\xi, \eta \in \mathbb{C}$ and any value of the parameter $\lambda \in \mathbb{C}$, there exists a unique solution of the differential equation (2.2), say $y(\cdot, \lambda):(a, b) \rightarrow \mathbb{C}$, with the properties:

$$
\begin{cases}(i) & y(\cdot, \lambda) \text { and }\left(p y^{\prime}\right)(\cdot, \lambda) \in A C_{\mathrm{loc}}(a, b) \\ (\text { ii }) & y(c, \lambda)=\xi \text { and }\left(p y^{\prime}\right)(c, \lambda)=\eta \\ (\text { iii }) & y(x, \cdot) \text { and }\left(p y^{\prime}\right)(x, \cdot) \text { are holomorphic on } \mathbb{C} \\ & \text { for all } x \in(a, b) \\ (\text { iv }) \quad \text { if } \xi, \eta \in \mathbb{R}, \text { then } \bar{y}(\cdot, \lambda)=y(\cdot, \bar{\lambda}) \text { and } \\ \\ \left(p \bar{y}^{\prime}\right)(\cdot, \lambda)=\left(p y^{\prime}\right)(\cdot, \bar{\lambda}) \text { for all } \lambda \in \mathbb{C} .\end{cases}
$$


Green's formula for the differential expression $M$ is, for any compact interval $[\alpha, \beta] \subset(a, b)$,

$$
\int_{\alpha}^{\beta}\{\bar{g}(x) M[f](x)-f(x) \overline{M[g]}(x)\} d x=[f, g](\beta)-[f, g](\alpha) \text { for all } f, g \in D(M),
$$

where the symplectic form $[\cdot, \cdot](x): D(M) \times D(M) \rightarrow \mathbb{C}$ is defined by

$$
[f, g](x):=f(x)\left(p \bar{g}^{\prime}\right)(x)-\left(p f^{\prime}\right)(x) \bar{g}(x) \text {, for each } x \in(a, b) .
$$

The analysis of boundary value problems for the differential expression (2.4) takes place in the weighted Hilbert function space $L^{2}((a, b) ; w)$ with norm and scalar product given by

$$
\|f\|_{w}^{2}=\int_{a}^{b} w(x)|f(x)|^{2} d x \text { and }(f, g)_{w}=\int_{a}^{b} w(x) f(x) \bar{g}(x) d x .
$$
by

$$
D\left(T_{1}\right):=\left\{f \in D(M): f, w^{-1} M[f] \in L^{2}((a, b) ; w)\right\}
$$

and

$$
T_{1} f:=w^{-1} M[f] \text { for all } f \in D\left(T_{1}\right) .
$$

From Green's formula (2.6) it follows that the two limits

$$
\lim _{x \rightarrow b}[f, g](x) \text { and } \lim _{x \rightarrow a}[f, g](x)
$$

both exist and are finite in $\mathbb{C}$, for all $f, g \in D\left(T_{1}\right)$.

The minimal operator $T_{0}$ generated by the expression $M$ in this space is defined by the dense domain $D\left(T_{0}\right) \subset L^{2}((a, b) ; w)$ :

$$
D\left(T_{0}\right):=\left\{f \in D\left(T_{1}\right): \lim _{x \rightarrow b}[f, g](x)-\lim _{x \rightarrow a}[f, g](x)=0 \text { for all } g \in D\left(T_{1}\right)\right\}
$$

and

$$
T_{0} f:=w^{-1} M[f] \text { for all } f \in D\left(T_{0}\right) .
$$

From established results in [27, Chapter V], 9], 10, Appendix A] and [11, Appendix] it is known that the following properties of $T_{0}$ and $T_{1}$ hold, where ${ }^{*}$ denotes an adjoint operator in $L^{2}((a, b) ; w)$ :

$$
\begin{cases}(\text { i }) & D\left(T_{0}\right) \subseteq D\left(T_{1}\right) \text { and } T_{0} \subseteq T_{1} \\ (\text { ii }) & T_{0} \text { is a closed, symmetric operator in } L^{2}((a, b) ; w) \\ \text { (iii) } & T_{1} \text { is a closed operator in } L^{2}((a, b) ; w) \\ \text { (iv }) & T_{0}^{*}=T_{1} \text { and } T_{1}^{*}=T_{0} .\end{cases}
$$

The deficiency indices $\left\{d^{-}, d^{+}\right\}$of the operator $T_{0}$ (see Example 1.13) are equal since the coefficients $p, q, w$ are all real-valued on $(a, b)$. Since the differential expression $M$ is of the second-order, it follows that, defining the common index $d$,

$$
d:=d^{-}=d^{+}=0,1 \text { or } 2 .
$$

The integer value of this common deficiency index depends upon the properties of the three coefficients $p, q, w$ at the endpoints $a$ and $b$ of the interval $(a, b)$, i.e. the classification of these endpoints as regular, limit-circle or limit-point in the space $L^{2}((a, b) ; w)$; see [5, Chapter 9], [7] and [32, Chapter II]. 
We interpret the boundary value problem for a symmetric linear differential expression, or formal differential operator $M$ (for instance, the Sturm-Liouville expression $M$ on its domain $D(M)$ in the Hilbert space $L^{2}((a, b) ; w)$ as in (2.3), (2.4) and (2.8)), to be the problem of determining all self-adjoint linear operators $T$ generated by $M$ on the relevant Hilbert space; i.e. $T$ on the domain $D(T)$ is an extension of the minimal operator $T_{0}$ on $D\left(T_{0}\right)$ and a restriction of the maximal operator $T_{1}$ on $D\left(T_{1}\right)$. Then further we seek to examine whether each such selfadjoint operator $T$ on $D(T)$ can be defined by imposing boundary conditions on the functions of $D\left(T_{1}\right)$ - and how this action can be accomplished. In this sense, the classical eigenfunction expansions (or more general integral representations for functions of the specified Hilbert space) are now incorporated into the spectral theory of self-adjoint linear operators.

To discuss all the self-adjoint extensions $\{T\}$ of the symmetric operator $T_{0}$, and thereby introduce the connections between the Sturm-Liouville boundary value problems of the differential equation (2.2) and complex symplectic spaces, we define the symplectic form $[\cdot, \cdot]: D\left(T_{1}\right) \times D\left(T_{1}\right) \rightarrow \mathbb{C}$ by (see (2.11) $)$

$$
[f, g]:=\lim _{x \rightarrow b}[f, g](x)-\lim _{x \rightarrow a}[f, g](x) \text { for all } f, g \in D\left(T_{1}\right) .
$$

We note that, from (2.12),

$$
[f, g]=0 \text { for all } f \in D\left(T_{1}\right) \text { and all } g \in D\left(T_{0}\right) .
$$

Define the complex symplectic space $S$ for the symmetric operator $T_{0}$ (see (1.51) and (1.57)) as the quotient space of cosets

$$
\mathrm{S}:=D\left(T_{1}\right) / D\left(T_{0}\right) \text {. }
$$

Note that from the formulae (1.45), (1.47) and (2.15) we obtain the result

$$
\operatorname{dim}(\mathrm{S})=2 d \text {. }
$$

Writing now the elements of this space $\mathrm{S}$, using the notation $\mathrm{f}=\left\{f+D\left(T_{0}\right)\right\}$ and $\mathrm{g}=\left\{g+D\left(T_{0}\right)\right\}$, the symplectic product $[\cdot: \cdot]$ on $\mathrm{S}$ is given by (see (1.53)):

$$
[\mathrm{f}: \mathrm{g}]:=[f, g]
$$

where the right-hand side is defined from (2.16), using the representative functions $f \in \mathrm{f}, g \in \mathrm{g}$.

When the common deficiency index $d=0$ it follows that there is a unique selfadjoint operator $T=T_{0}=T_{0}^{*}=T_{1}$, that the complex symplectic space $\mathrm{S}$ has dimension zero, and that no boundary conditions need to be imposed upon the domain $D\left(T_{1}\right)$ to give the self-adjoint operator $T$.

That said, we now suppose that $d=1$ or $d=2$ holds. In this case the complex symplectic space $\mathrm{S}$ has even integer finite dimension; thus the results of 10 , Section III, Theorem 2 and Corollary 2] and [14, Section 2, Theorem 2.3] apply, and further for this space $\mathrm{S}$ we have (see (1.61))

$$
\mathbf{p}=\mathbf{q}
$$

The following results then hold:

(i) if $d=1$ there exist complete Lagrangian subspaces $\mathrm{L}$, all with $\operatorname{dim}(\mathrm{L})=1$

(ii) if $d=2$ there exist complete Lagrangian subspaces $\mathrm{L}$, all with $\operatorname{dim}(\mathrm{L})=2$. 
For the remainder of this section we continue this discussion for the SturmLiouville boundary value problems but use a notation which is also valid for the corresponding boundary value theory of Lagrange symmetric ordinary differential expressions of higher order $n \geq 2$, with $\operatorname{dim}(\mathrm{S})=2 d \leq 2 n$. This subject is treated in greater detail and generality in Section 4.2

We now call on the GKN-Theorem; see Theorem [1.14, [27, Chapter V] or the more general account in 9. This theorem states that for every Sturm-Liouville self-adjoint extension $T$ of $T_{0}$ (restriction of $T_{1}$ ) there exists a set $\left\{w_{r} \in D\left(T_{1}\right)\right.$ : $1 \leq r \leq d\}$ having the properties:

( $i)$ the set $\left\{w_{r} \in D\left(T_{1}\right): 1 \leq r \leq d\right\}$ is linearly independent in $D\left(T_{1}\right)$ modulo $D\left(T_{0}\right)$

(ii) using the definition (2.16)

$$
\left[w_{r}, w_{s}\right]=0 \text { for } 1 \leq r, s \leq d,
$$

such that for the self-adjoint operator $T$

$$
\begin{aligned}
D(T) & =\left\{f \in D\left(T_{1}\right):\left[f, w_{r}\right]=0 \text { for } 1 \leq r \leq d\right\} \\
T f & =T_{1} f=w^{-1} M[f] \text { for all } f \in D(T) .
\end{aligned}
$$

The set $\left\{w_{r} \in D\left(T_{1}\right): 1 \leq r \leq d\right\}$ is called a GKN symmetric set of boundary condition functions; see [9].

Notation 2.1. Note that we use the symbol $w$ for:

( $i$ ) the weight coefficient in the differential equation (2.2) and in the weighted Hilbert function space $L^{2}((a, b) ; w)$ (2.8) as standard notation for SturmLiouville theory

(ii) the boundary condition functions in the set $\left\{w_{r} \in D\left(T_{1}\right): 1 \leq r \leq d\right\}$ to follow the standard notations in Naimark 27, Chapter V, Section 18.1, Theorem 4].

The context in which this symbol $w$ is used should indicate which one of these two cases is involved.

There is a converse to the above result: if there exists a set $\left\{w_{r} \in D\left(T_{1}\right): 1 \leq\right.$ $r \leq d\}$ satisfying the stated linear independence and the property given by item $(\mathrm{ii})$ above, then the operator $T$ defined by (2.24) and (2.25) is a self-adjoint extension of $T_{0}$ (restriction of $T_{1}$ ) having the indicated GKN symmetric set of boundary condition functions.

We can now identify the complete Lagrangian $\mathrm{L} \subset \mathrm{S}$ (see (2.22) ) with the linear manifold $D(T) \subset D\left(T_{1}\right)$, using Notation 1.3 and Theorem 1.14, by the identification

$$
\mathrm{w}_{r}=\left\{w_{r}+D\left(T_{0}\right)\right\} \text { for } 1 \leq r \leq d
$$

to give $\left\{\mathrm{w}_{r} \in \mathrm{S}: 1 \leq r \leq d\right\}$ as a linearly independent basis for $\mathrm{L}$ satisfying (see (2.20)

$$
\left[\mathrm{w}_{r}: \mathrm{w}_{s}\right]=0 \text { for } 1 \leq r, s \leq d .
$$

Again, as with the GKN formulation, there is a converse to this last result: if a complete Lagrangian $\mathrm{L} \subset \mathrm{S}$, necessarily with $\operatorname{dim}(\mathrm{L})=d$, can be found, then any basis $\left\{\mathrm{w}_{r} \in \mathrm{L}: 1 \leq r \leq d\right\}$ of $\mathrm{L}$ satisfies (2.27) and the identification (2.26) gives a GKN symmetric set of boundary condition functions with which to define $D(T)$ as in (2.24), as is consistent with the general result given in Remark 1.15. 
This account is an abstract formulation of boundary value problems for the Sturm-Liouville differential equation, given by (2.1) and (2.2), to connect the GKN theory [9], as also dependent upon the Stone-von Neumann theory of linear operators in abstract Hilbert space [31, with the properties of complex symplectic spaces and their complete Lagrangian subspaces. In Section 4 we give more explicit details of the structure of symmetric Sturm-Liouville boundary conditions and related results for higher-order quasi-differential expressions.

2.2. Laplace boundary value problems. In this section we illustrate an application of symplectic algebra to boundary value theory for an elliptic partial differential operator; see 13 and 34 for a full exposition of the general theory of second-order (and higher-order) elliptic partial differential expressions in bounded regions of $r$-dimensional real Euclidean space $\mathbb{E}^{r}$, for $r \geq 2$.

Consider the classical Laplace partial differential expression

$$
\Delta=\frac{\partial^{2}}{\partial x_{1}^{2}}+\frac{\partial^{2}}{\partial x_{2}^{2}}
$$

in terms of the rectangular cartesian co-ordinates $\left(x_{1}, x_{2}\right)$ within a bounded region $\Omega \subset \mathbb{E}^{2}$. That is, $\Omega$ is an open connected set in the Euclidean plane with a compact boundary $\partial \Omega$ consisting of smooth closed curves. For simplicity we take $\Omega$ to be the open unit disk

$$
\Omega=\left\{\mathbf{x}=\left(x_{1}, x_{2}\right) \in \mathbb{E}^{2}: 0 \leq x_{1}^{2}+x_{2}^{2}<1\right\},
$$

so $\partial \Omega$ is the unit circle.

Then $\Delta$ defines a linear operator $A$ on its classical domain $D(A)$ in the Hilbert space $L^{2}(\Omega)$; that is, with a conventional negative sign,

$$
\begin{gathered}
D(A):=C_{0}^{\infty}(\Omega) \\
A f:=-\Delta f \text { for all } f \in D(A) .
\end{gathered}
$$

Here the complex Hilbert function space

$$
L^{2}(\Omega)=\left\{f: \Omega \rightarrow \mathbb{C}: \int_{\Omega}|f(\mathbf{x})|^{2} d \mathbf{x}<\infty\right\}
$$

has the usual scalar product and norm, respectively, given by

$$
\langle f, g\rangle=\int_{\Omega} f(\mathbf{x}) \bar{g}(\mathbf{x}) d \mathbf{x} \text { and }\|f\|^{2}=\langle f, f\rangle,
$$

where $d \mathbf{x}=d x_{1} d x_{2}$ indicates Lebesgue measure in $\mathbb{E}^{2}$.

We recall that

$$
\left\{\begin{array}{r}
C^{\infty}(\Omega)=\{f: \Omega \rightarrow \mathbb{C}: \text { all classical derivatives of } f \\
\text { are continuous in } \Omega\}
\end{array}\right.
$$

and

$$
C_{0}^{\infty}(\Omega)=\left\{f \in C^{\infty}(\Omega): f \text { has compact support in } \Omega\right\} ;
$$

thus $D(A)$ (see (2.30) ) is a dense linear manifold of $L^{2}(\Omega)$. Moreover, the classical Green formula, for all complex-valued suitably smooth functions $f, g$ on the closed $\operatorname{disk} \bar{\Omega}$, asserts

$$
\langle-\Delta f, g\rangle-\langle f,-\Delta g\rangle=\int_{\partial \Omega}\left\{\frac{\partial f}{\partial \mathbf{n}} \bar{g}-f \frac{\partial \bar{g}}{\partial \mathbf{n}}\right\} d \sigma
$$


with inwards unit normal $\mathbf{n}$ on $\partial \Omega$ and arc length $d \sigma$ on $\partial \Omega$. Hence, for $f, g \in D(A)$ as in (2.30), it is straightforward to calculate (using the continuous extensions so $f=g=0$ on $\partial \Omega)$,

$$
\langle A f, g\rangle-\langle f, A g\rangle=0
$$

so $A$ on $D(A)$ is a symmetric operator in $L^{2}(\Omega)$.

However, $A$ on $D(A)$ is not self-adjoint (not even closed), so we have to obtain its closure and then determine the corresponding minimal and maximal operators (compare with Section 2.1), respectively, on the domains in $L^{2}(\Omega)$,

$$
T_{0} \text { on } D\left(T_{0}\right) \text { and } T_{1} \text { on } D\left(T_{1}\right),
$$

in order to apply the general constructions in Example 1.13 in Section 1, For this purpose we require the Sobolev spaces, which we regard as submanifolds of $L^{2}(\Omega)$, say

$$
W^{2}(\Omega) \subset W^{1}(\Omega) \subset L^{2}(\Omega),
$$

as defined next; see [13, Appendix A].

2.2.1. Notation for Sobolev spaces. Consider the linear submanifolds of $L^{2}(\Omega)$ given by

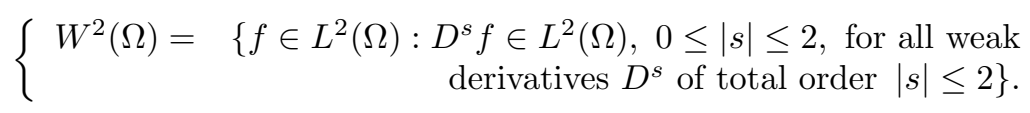

Here, for non-negative integers $s_{1}, s_{2}$ and $|s|=s_{1}+s_{2}$,

$$
D^{s} f=\frac{\partial^{|s|} f}{\partial x_{1}^{s_{1}} \partial x_{2}^{s_{2}}},
$$

are weak or distributional derivatives of $f \in L^{2}(\Omega)$. Then $W^{2}(\Omega)$ is a (Sobolev) Hilbert space with the scalar product and norm given by, for all $f, g \in W^{2}(\Omega)$,

$$
\langle f, g\rangle_{2}=\sum_{0 \leq|s| \leq 2}\left\langle D^{s} f, D^{s} g\right\rangle \text { and }\|f\|_{2}=\left|\langle f, f\rangle_{2}\right|^{1 / 2} .
$$

By the Trace Theorem [13, Appendix A], upon using appropriate limits, $f \in W^{2}(\Omega)$ assigns the values of both $f$ and the normal derivative $\partial f / \partial \mathbf{n}$ on $\partial \Omega$ :

$$
\left.f\right|_{\partial \Omega} \text { and }\left.\frac{\partial f}{\partial \mathbf{n}}\right|_{\partial \Omega}
$$

both in the space $L^{2}(\partial \Omega)$. In particular, we denote the closed Hilbert subspace of $W^{2}(\Omega)$

$$
\stackrel{\circ}{W}^{2}(\Omega)=\left\{f \in W^{2}(\Omega): f=0 \text { and } \frac{\partial f}{\partial \mathbf{n}}=0 \text { on } \partial \Omega\right\} .
$$

In a similar way we define the (Sobolev) Hilbert spaces $W^{1}(\Omega)$ and $\stackrel{\circ}{W^{1}}(\Omega)$; thus, in particular,

$$
W^{2}(\Omega) \cap \stackrel{\circ}{W}{ }^{1}(\Omega)=\left\{f \in W^{2}(\Omega): f=0 \text { on } \partial \Omega\right\} .
$$

We now return to Section 2.2 and to the study of the Laplace differential expression $\Delta$ of (2.28). Using the definitions above, we are prepared to follow the abstract framework of Example 1.13 and define the corresponding minimal and 
maximal operators $T_{0}$ and $T_{1}$, respectively, for the operator $A$ on $D(A)$; see (2.38). Thereafter we define the boundary complex symplectic space

$$
\mathrm{S}:=D\left(T_{1}\right) / D\left(T_{0}\right)
$$

in order to use both the GKN-Theorem and the structure of the complete Lagrangian subspaces $\mathrm{L} \subset \mathrm{S}$ to determine all the self-adjoint extensions of the operator $A=-\Delta$ of (2.31) through these methods.

First we remark that $f, g \in W^{2}(\Omega)$ satisfy Green's formula (2.36), upon using weak derivatives for the Laplacian and the appropriate trace values for the data on the boundary $\partial \Omega$. Hence Green's formula, a fortiori, applies to $f, g \in \stackrel{\circ}{W^{2}}(\Omega)$, and we define the extension of $A$ to $T_{0}$ by

$$
D\left(T_{0}\right):=\stackrel{\circ}{W}{ }^{2}(\Omega) \text { and } T_{0} f:=-\Delta f \text { for all } f \in D\left(T_{0}\right) .
$$

Then $T_{0}$ is the (closure) minimal closed symmetric extension of $A$ on $D(A)$ in the Hilbert space $L^{2}(\Omega)$; see [13, Theorem 3.2].

The corresponding maximal operator $T_{1}$, as generated by $A$ on $D(A)$, is defined to be the $L^{2}(\Omega)$-adjoint of $T_{0}$ :

$$
T_{1}:=T_{0}^{*} \text { on } D\left(T_{1}\right) \supset D\left(T_{0}\right) .
$$

It has been established (see [13, Theorem 3.3 and Example 4.1] and 30, Volume 5, Section 188]) that $D\left(T_{1}\right)$ is the direct sum of two submanifolds of $L^{2}(\Omega)$, namely

$$
W^{2}(\Omega) \cap \stackrel{\circ}{W}{ }^{1}(\Omega):=\left\{f \in W^{2}(\Omega): f=0 \text { on } \partial \Omega\right\}
$$

and

$$
\stackrel{\Delta}{L}^{2}(\Omega):=\left\{f \in L^{2}(\Omega): f \in C_{0}^{\infty}(\Omega) \text { and } \Delta f \equiv 0 \text { in } \Omega\right\} .
$$

That is, we define the submanifold of $L^{2}(\Omega)$

$$
\stackrel{\Delta}{W}^{2}(\Omega):=W^{2}(\Omega) \cap \stackrel{\circ}{W}{ }^{1}(\Omega) \dot{+} \stackrel{\Delta}{l}^{2}(\Omega)
$$

(see [13, Lemma 3.2]) to obtain also

$$
\stackrel{\Delta}{W}^{2}(\Omega)=\operatorname{span}\left\{W^{2}(\Omega), \stackrel{\Delta}{L}{ }^{2}(\Omega)\right\} .
$$

Here $W^{2}(\Omega) \cap \stackrel{\circ}{W}{ }^{1}(\Omega)$ is a submanifold of $L^{2}(\Omega)$ which contains $\stackrel{\circ}{W}{ }^{2}(\Omega)$ (see (2.43) and (2.44) ), and $\stackrel{\Delta}{L}^{2}(\Omega)$ consists of all complex-valued harmonic functions that belong to $L^{2}(\Omega)$.

While $\stackrel{\Delta}{L^{2}}(\Omega) \subset L^{2}(\Omega)$ we observe that $\stackrel{\Delta}{L}^{2}(\Omega) \varsubsetneqq W^{2}(\Omega)$; see the example in Section 2.3 . Accordingly we seek to extend $A=-\Delta$ on $D(A)$ to $\stackrel{\Delta}{W}^{2}(\Omega)$ by using weak derivatives on $W^{2}(\Omega)$ and the structure of the direct sum (2.50). Namely, each function $f \in \vec{W}^{2}(\Omega)$ has a unique representation

$$
f=f_{D}+f_{\Delta}, \text { with } f_{D} \in W^{2}(\Omega) \cap \stackrel{\circ}{W}(\Omega) \text { and } f_{\Delta} \in \stackrel{\Delta}{L}(\Omega) .
$$

In this situation we define

$$
-\Delta f:=-\Delta f_{D}\left(\text { noting that } \Delta f_{\Delta}=0\right) .
$$


Therefore we now obtain the required maximal operator $T_{1}$ on $D\left(T_{1}\right)$, as generated by $A$ on $D(A)$, namely

$$
D\left(T_{1}\right):=\stackrel{\Delta}{W}^{2}(\Omega) \text { and } T_{1} f:=-\Delta f_{D} \text { for all } f \in D\left(T_{1}\right) .
$$

These results lead to the correct definition of the boundary complex symplectic space $\mathrm{S}$ for the operator $A=-\Delta$ on $D(A) \subset L^{2}(\Omega)$; that is

$$
\mathrm{S}:=D\left(T_{1}\right) / D\left(T_{0}\right) \text {, }
$$

with the symplectic product

$$
[\mathrm{f}: \mathrm{g}]:=[f+\stackrel{\mathrm{o}}{W}(\Omega): g+\stackrel{\mathrm{o}}{W}(\Omega)]:=\int_{\Omega}\{(-\Delta f) \bar{g}-f(-\Delta \bar{g})\} d \mathbf{x},
$$

where $f, g \in \stackrel{\Delta}{W}^{2}(\Omega)$ represent the cosets, respectively,

$$
\mathrm{f}=\left\{f+\stackrel{\circ}{W}{ }^{2}(\Omega)\right\} \text { and } \mathrm{g}=\left\{g+\stackrel{\circ}{W^{2}}(\Omega)\right\},
$$

as in Example 1.13 of Section 1,

We are now in a position to describe all the self-adjoint operators $T$ on domains $D(T)$, as extensions of $A=-\Delta$ on $D(A)$, by means of the GKN-EM Theorem 1.14. First note that $\Delta$ is a real operator so the deficiency indices of the minimal operator $T_{0}$ (equivalently the negativity and positivity indices of $\mathrm{S}$ ) are equal:

$$
d^{-}=d^{+}=d \text { (say), equivalently } \mathbf{q}=\mathbf{p},
$$

and moreover $d$ is the cardinality of $\mathbb{N}$, that is

$$
d=\aleph_{0}
$$

further this result has the interesting consequence

$$
\operatorname{dim}(\mathrm{S})=2 d=\aleph_{0} .
$$

Hence, there exists a non-countable set $\{T\}$ of such self-adjoint operators

$$
T_{0} \subset T=T^{*} \subset T_{1} \text { with domains } \stackrel{\circ}{W}{ }^{2}(\Omega) \subset D(T)=D\left(T^{*}\right) \subset \stackrel{\Delta}{W}^{2}(\Omega) .
$$

As an example, consider the classical Dirichlet self-adjoint operator $T_{\mathrm{Dir}}$ on the domain (see [13, Definition 4.1 and Theorem 4.1])

$$
T_{\text {Dir }} f=-\Delta f \text { on } D\left(T_{\text {Dir }}\right)=W^{2}(\Omega) \cap \stackrel{\circ}{W}{ }^{1}(\Omega)
$$

corresponding to the Dirichlet complete Lagrangian (see Theorem 1.14)

$$
\mathrm{L}_{\text {Dir }}=\Psi\left(W^{2}(\Omega) \cap \stackrel{\circ}{W}(\Omega)\right) \subset \mathrm{S},
$$

in terms of the natural projection map $\Psi$ (see (1.52) and Remark 1.15 and note that $\stackrel{\circ}{W}^{2}(\Omega) \subset W^{2}(\Omega) \cap \stackrel{\circ}{W^{1}}(\Omega)$ which implies $\stackrel{\circ}{W^{2}}(\Omega)$ is contained in $\left.D\left(T_{\text {Dir }}\right)\right)$.

As is well-known, the Dirichlet operator $T_{\text {Dir }}$ has a discrete spectrum of real eigenvalues (with each eigenspace of finite dimension), with no accumulation points in $\mathbb{R}$. However, a more unusual self-adjoint extension of $A=-\Delta$ on $D(A)=C_{0}^{\infty}(\Omega)$ is the harmonic operator $T_{\mathrm{Har}}$ (see [15])

$$
D\left(T_{\mathrm{Har}}\right):=\stackrel{\circ}{W}{ }^{2}(\Omega)+\stackrel{\Delta}{L}^{2}(\Omega) \text { and } T_{\mathrm{Har}} f:=-\Delta f \text { for all } f \in D\left(T_{\mathrm{Har}}\right),
$$


with the corresponding complete Lagrangian

$$
\mathrm{L}_{\mathrm{Har}}:=\Psi\left(\stackrel{\Delta}{L}^{2}(\Omega)\right) \subset \mathrm{S} .
$$

This self-adjoint operator $T_{\text {Har }}$ has a spectrum which includes a countable set of discrete eigenvalues, each of finite multiplicity, but with a non-empty essential spectrum consisting of a single eigenvalue at the origin of infinite multiplicity; see [15].

2.3. Comparison of boundary value problems. Finally we compare and contrast the boundary value theory for ordinary differential operators (as exemplified by the Sturm-Liouville operators in Section 2.1) and for elliptic partial differential operators (as exemplified by the Laplace operator in Section 2.2), especially as illuminated through the methods of symplectic algebra.

Of course, the boundary complex symplectic space $\mathrm{S}$ is finite dimensional in the Sturm-Liouville case and infinite dimensional for the Laplace operator. Otherwise the GKN-Theorem applies equally well to correlate the self-adjoint extensions to the complete Lagrangians of $\mathrm{S}$. For the finite dimensional case a Lagrangian subspace $L \subset S$ is complete if and only if $2 \operatorname{dim}(L)=\operatorname{dim}(S)$, whereas for the infinite dimensional case this (cardinal) arithmetic condition is necessary but not sufficient for $L$ to be complete; compare (2.58).

The boundary conditions which define a self-adjoint extension for a SturmLiouville operator can always be formulated in terms of linear homogeneous conditions on the boundary value of functions and their first derivatives (or limits of such conditions in singular cases). However the operator $T_{\mathrm{Har}}$ on $D\left(T_{\mathrm{Har}}\right)$, for the Laplace expression, is not subject to characterization by conditions for functions described by data on the boundary circle $\partial \Omega$ of the disk $\Omega \subset \mathbb{E}^{2}$. However, it should be pointed out that, even for partial differential expressions, the GKN-Theorem does permit a characterization of the corresponding complete Lagrangian subspace $\mathrm{L} \subset \mathrm{S}$ by means of a system of linear functionals on $\mathrm{S}$, each of which is specified by a vector of a basis of $\mathrm{L}$; see [14, Proposition 5.1] and compare (2.24).

The operator $T_{\mathrm{Har}}$ on $D\left(T_{\mathrm{Har}}\right) \subset L^{2}(\Omega)$ seems rather mysterious, especially if we consider the Laplace operator on the bounded region $\Omega \subset \mathbb{E}^{2}$ as the analogue of a regular boundary value problem for the Sturm-Liouville operator.

For instance, the domain

$$
D\left(T_{\text {Har }}\right)=\stackrel{\circ}{W}{ }^{2}(\Omega)+\stackrel{\Delta}{L}^{2}(\Omega)
$$

does not lie within the domain $W^{2}(\Omega)$, which might be expected to play the role of the maximal domain. An explicit example of an element $F \in D\left(T_{\mathrm{Har}}\right)$ demonstrates this property (see [15. Example 4.2]), as given next. Namely, consider the analytic function

$$
F(z):=\sum_{n=0}^{\infty} z^{2^{n}} \text { for all }|z|<1,
$$

which is holomorphic for this open unit disk of $\mathbb{C}$ or $\mathbb{E}^{2}$ (using the complex coordinate $z=r \exp (i \theta)$, as usual). It is straightforward to show that $F \in L^{2}(\Omega)$, and hence, since $F$ is harmonic, $F \in \stackrel{\Delta}{L}^{2}(\Omega)$. Clearly on the unit circle $z=\exp (i \theta)$, the 
infinite series (2.64) converges nowhere. Indeed, computations for $F^{\prime}$ given by

$$
F^{\prime}(z)=\sum_{n=0}^{\infty} 2^{n} z^{2^{n}-1} \text { for all }|z|<1
$$

show that

$$
F^{\prime} \notin L^{2}(\Omega) \text { so that } F \notin W^{1}(\Omega),
$$

and, significantly, the Trace Theorem (see [15, Remark 4.4]) does not apply and hence assigns no boundary values for $F$ on the unit circle, the boundary $\partial \Omega$ of $\Omega$.

In a desperate attempt to assign some reasonable boundary data to the function $F$ on the boundary $\partial \Omega$, we could examine radial limits, for $\theta \in[0,2 \pi)$, say

$$
\lim _{r \nearrow 1} F(r \exp (i \theta))=\lim _{r \nearrow 1} \sum_{n=0}^{\infty} r^{2^{n}} \exp \left(i 2^{n} \theta\right) .
$$

But again more difficult analysis (see [15. Example 4.2]) proves that, for each $\theta \in$ $[0,2 \pi)$, the required finite radial limit, as $r \nearrow 1$, does not exist.

As another example, the analytic function $G$ (see [15, (4.24)]),

$$
G(z)=\sum_{n=0}^{\infty} n z^{2^{n}} \text { for all }|z|<1
$$

belongs to $\stackrel{\Delta}{L}^{2}(\Omega)$, and furthermore, for each ray given by $\theta \in[0,2 \pi)$,

$$
\limsup _{r \nearrow 1}|G(r \exp (i \theta))|=+\infty .
$$

As before,

$$
G^{\prime} \notin L^{2}(\Omega) \text { so that } G \notin W^{1}(\Omega) .
$$

Another interesting and strange property of $T_{\text {Har }}$ on $D\left(T_{\text {Har }}\right)$ is that (see Section 2.2) the essential spectrum is non-empty, in contrast to the discrete spectrum for all regular Sturm-Liouville problems. It is an unsolved problem as to whether the operator $T_{\text {Har }}$, as a self-adjoint extension for the Laplace operator in the unit disk, is unique in some noteworthy way. For instance, can $T_{\mathrm{Har}}$ be characterized, among all self-adjoint operators for the Laplace differential expression or even among some interesting subclass, by mathematically significant intrinsic properties?

The question of uniqueness for the operator $T_{\mathrm{Har}}$ also leads to another problem. If $T_{\text {Har }}$ is unique, can all other self-adjoint extensions of the Laplace operator in the unit disk be determined by applying generalized boundary conditions to functions in the maximal domain $D\left(T_{1}\right)$, on the boundary $\partial \Omega$, using the properties of the Trace Theorem?

\section{General theory of COMPlex sympleCtic SPACES}

Recall Definition 1.1 of a complex symplectic space S, with symplectic product $[\cdot ; \cdot]$, say of finite or infinite (non-finite) dimension over the complex field $\mathbb{C}$. With full generality in Section 1, we have formally specified two kinds of intrinsic structures for $\mathbf{S}$, namely the (cardinal) numeric invariants of the positivity index $\mathbf{p}$, negativity index $\mathbf{q}$, and Lagrangian index $\Delta=\min \{\mathbf{p}, \mathbf{q}\}$, as in Definition 1.11 and the topological properties ascribed to the symplectic weak topology, as in Definition 1.12. We should also observe here that $\mathrm{S}$, together with the conjugate bilinear form $-i[\cdot: \cdot]$, is a (non-degenerate) indefinite inner-product space, and conversely every 
such indefinite inner-product space defines a corresponding complex symplectic space; see [3] and [14].

While these concepts are meaningful for each complex symplectic space $\mathrm{S}$, they are more readily interpreted and explicitly useful in case $S$ has a Hilbert structure, say as exhibited by a Hilbert pair $\left\{\mathrm{H}_{ \pm}\right\}$; see Example 1.9. In this case we assume there exists a symplectically orthogonal direct sum decomposition

$$
\mathrm{S}=\mathrm{H}_{-} \oplus \mathrm{H}_{+}
$$

that is let $\mathrm{H}_{-}$and $\mathrm{H}_{+}$be complex Hilbert spaces (finite or infinite dimensional), with corresponding scalar products and norms $\langle\cdot, \cdot\rangle_{ \pm}$and $\|\cdot\|_{ \pm}$, respectively, so that each vector $\mathrm{u} \in \mathrm{S}$ has a unique representation as an ordered couple

$$
\mathrm{u}=\left(\mathrm{u}_{-}, \mathrm{u}_{+}\right) \text {, with } \mathrm{u}_{-} \in \mathrm{H}_{-} \text {and } \mathrm{u}_{+} \in \mathrm{H}_{+},
$$

and the vectors $\left(\mathbf{u}_{-}, 0\right)$ and $\left(0, \mathbf{u}_{+}\right)$are orthogonal in $\mathbf{S}$. Then we have

$$
\left\langle\mathrm{u}_{-}, \mathrm{v}_{-}\right\rangle_{-}=i\left[\left(\mathrm{u}_{-}, 0\right):\left(\mathrm{v}_{-}, 0\right)\right] \text { and }\left\langle\mathrm{u}_{+}, \mathrm{v}_{+}\right\rangle_{+}=-i\left[\left(0, \mathrm{u}_{+}\right):\left(0, \mathrm{v}_{+}\right)\right] .
$$

Indeed, $\mathrm{S}$ can be treated as the direct sum Hilbert space with norm (but not the topology; see (3.18)) dependent on the Hilbert pair $\left\{\mathrm{H}_{ \pm}\right\}$.

This Hilbert structure is always the case when $\operatorname{dim}(\mathrm{S})<+\infty$, and then (see Example 1.8)

$$
\mathbf{q}=\operatorname{dim}\left(\mathbf{H}_{-}\right) \text {and } \mathbf{p}=\operatorname{dim}\left(\mathbf{H}_{+}\right)
$$

(independent of the choice of the Hilbert pair $\left\{\mathrm{H}_{ \pm}\right\}$decomposing $\mathrm{S}$ ). Moreover,

$$
\{\mathbf{p}, \mathbf{q}\}
$$

constitutes a complete set of invariants for $\mathbf{S}$ up to symplectic isomorphism, and we have the following results:

$$
\Delta=\min \{\mathbf{p}, \mathbf{q}\}=\max \{\operatorname{dim}(\mathrm{L}): \mathrm{L} \text { is a Lagrangian subspace of } \mathrm{S}\} .
$$

In addition there exists a complete Lagrangian $\mathrm{L} \subset \mathrm{S}$ if and only if

$$
\mathbf{p}=\mathbf{q}
$$

that is if and only if

$$
\operatorname{Ex}(\mathrm{S})=\mathbf{p}-\mathbf{q}=0
$$

also a Lagrangian $\mathrm{L} \subset \mathrm{S}$ is complete if and only if

$$
2 \operatorname{dim}(\mathrm{L})=\operatorname{dim}(\mathrm{S}) \text {. }
$$

However, even if $\mathbf{S}$ is an infinite dimensional complex symplectic space with a Hilbert structure, as in (3.1), (3.2) and (3.3) (i.e. S corresponds to a Krein innerproduct space or to a Pontryagin space when, say, $\mathbf{q}<+\infty$ ), all the conclusions (3.4) to (3.8) remain valid (see [14, Section 4]), that is provided we interpret the invariants $\mathbf{p}$ and $\mathbf{q}$ as cardinal numbers, the dimension of any Hilbert space as the cardinality of an orthonormal basis, and in (3.7) the term $\operatorname{dim}(\mathrm{L})$ refers to closed Lagrangian subspaces in the Hilbert topology of S. The final assertion (3.9) is replaced by the statement that if a Lagrangian $\mathrm{L} \subset \mathrm{S}$ is complete, then

$$
2 \operatorname{dim}(\mathrm{L})=\operatorname{dim}(\mathrm{S})
$$

but the converse does not hold for infinite dimensional complex symplectic spaces. 
We note that for an infinite dimensional complex symplectic space $\mathrm{S}$ there may well be some simplifications of statements such as (3.6) to read:

$$
\operatorname{dim}(\mathrm{S})=\max \{\mathbf{p}, \mathbf{q}\},
$$

and for (3.10) to read that if a Lagrangian $\mathrm{L} \subset \mathrm{S}$ is complete, then

$$
\operatorname{dim}(\mathrm{L})=\mathbf{p}=\mathbf{q}=\operatorname{dim}(\mathrm{S}) .
$$

Also the proof of some of these statements can be quite difficult, for example the result (3.7). For an important case of these difficulties, see 14, Section 4, Theorem 4.3], the proof of which result is based on a transfinite version of the Gram-Schmidt process as given in [14, Section 4, Proposition 4.1]; see also [21.

Other differences between the cases of finite versus infinite dimensional complex symplectic spaces arise through investigation of the corresponding symplectic weak topologies. For instance, a complex symplectic space $\mathrm{S}$ is finite dimensional if and only if $\mathbf{S}$ is locally compact. We survey some of these topological contrasts below.

Finally, we remark that there exist infinite dimensional complex symplectic spaces $\mathrm{S}$ which admit no Hilbert structure, as in (3.1) - in fact, not even if preHilbert spaces $\left\{\mathrm{H}_{ \pm}\right\}$are allowed in the decomposition of S; see [3, Example 11.3]. These spaces are poorly understood; for instance, there appears to be no useful definition of $\operatorname{dim}(\mathrm{S})$ (the classical Hamel dimension does not seem relevant here). Nevertheless, even such an infinite dimensional complex symplectic space is a locally convex topological space, which is not however locally compact under its symplectic weak topology.

We next compare and contrast the properties of various topologies used on complex symplectic spaces. Let $\mathrm{S}$ be an arbitrary complex symplectic space, with a symplectic product [ : : ], as in Definition 1.1 of Section 11. Accordingly, the symplectic weak topology on $\mathrm{S}$ is specified in Definition 1.12 to be the (unique) weakest topology such that each linear functional $F_{\mathrm{w}}$, for w $\in \mathrm{S}$, is continuous:

$$
F_{\mathrm{w}}: \mathrm{S} \rightarrow \mathbb{C} \text { given by } \mathrm{u} \rightarrow[\mathrm{u}: \mathrm{w}] \text {. }
$$

Explicitly (3.13) demands that, for each open set $\mathcal{O} \subset \mathbb{C}$, the inverse image, for each $w \in S$,

$$
F_{\mathrm{w}}^{-1}(\mathcal{O}):=\{\mathrm{u} \in \mathrm{S}:[\mathrm{u}: \mathrm{w}] \in \mathcal{O}\}
$$

is weak-open in $\mathrm{S}$, and these weak-open sets then constitute a base for the symplectic weak topology. Moreover, we can take $\mathcal{O}$ to be an $\varepsilon$-disk, for each $\varepsilon>0$, say centered at a point $z_{0}=\left[\mathrm{u}_{0}: \mathrm{w}\right]$, for some choice of $\mathrm{u}_{0} \in \mathrm{S}$, and then

$$
F_{\mathrm{w}}^{-1}(\mathcal{O})=\left\{\mathrm{u} \in \mathrm{S}:\left|[\mathrm{u}: \mathrm{w}]-\left[\mathrm{u}_{0}: \mathrm{w}\right]\right|<\varepsilon\right\}
$$

is a weak-open neighborhood of $\mathrm{u}_{0}$ in $\mathrm{S}$. In this way we construct a useful neighborhood base for $u_{0} \in S$ by employing finite sets $W=\left\{w_{\alpha} \in S\right\}$, say for all $w_{\alpha} \in W$ :

$$
\mathrm{N}_{\mathrm{u}_{0}}(\mathrm{~W}, \varepsilon)=\left\{\mathrm{u} \in \mathrm{S}:\left|\left[\mathrm{u}: \mathrm{w}_{\alpha}\right]-\left[\mathrm{u}_{0}: \mathrm{w}_{\alpha}\right]\right|<\varepsilon\right\} .
$$

Since $\mathrm{S}$ is non-degenerate, i.e. for each pair of distinct points of $\mathrm{S}$ (say $\mathrm{u}_{1} \neq \mathrm{u}_{0}$ ), there exists $\mathrm{w}_{1} \in \mathrm{S}$ for which $\left|\left[\mathrm{u}_{1}-\mathrm{u}_{0}: \mathrm{w}_{1}\right]\right|>1$, and so the symplectic weak topology satisfies the Hausdorff separation axiom; see [22, Chapter 2, page 67]. Since each set $\mathrm{N}_{\mathrm{u}_{0}}(\mathrm{~W}, \varepsilon)$ of (3.16) is convex in the vector space $\mathrm{S}$, the symplectic weak topology establishes $\mathrm{S}$ as a locally convex topological vector space; for details see [14, Section 3]. 
A list of general properties of any locally convex vector space is provided in 14, Section 3, Remark 3.2], and important specific properties of S, with its symplectic weak topology, are developed in [14, Section 3, pages 23 and 24], for instance:

Remark 3.1. The following properties hold:

(1) $\mathbf{S}$ is finite dimensional if and only if $\mathbf{S}$ is locally compact.

(2) For each non-empty set $\mathrm{U} \subseteq \mathrm{S}$ the symplectic ortho-complement $\mathrm{U}^{\#}$ is closed.

(3) If $\mathrm{S}=\mathrm{S}_{1} \oplus \mathrm{S}_{2}$, then $\mathrm{S}_{1}=\mathrm{S}_{2}^{\#}$ and $\mathrm{S}_{2}=\mathrm{S}_{1}^{\#}$ are each complex symplectic subspaces and closed in S. Further, the intrinsic symplectic weak topologies in $S_{1}$ and $S_{2}$ coincide with the inherited or induced topologies from $S$.

(4) Each complete Lagrangian $L \subset S$ is closed in the symplectic weak topology of $\mathrm{S}$.

In certain special cases other topologies are relevant for the complex symplectic space S. For instance, if

$$
D:=\operatorname{dim}(\mathrm{S})<+\infty
$$

is finite, then $S$ is linearly isomorphic to the complex vector space $\mathbb{C}^{D}$ (as explicitly exhibited by the choice of a basis for S). In this way the classical Hermitian metric on $\mathbb{C}^{D}$ defines a metrizable topology (say, the Hermitian topology, independent of the choice of basis) establishing $\mathbf{S}$ as a locally compact topological vector space. Since a finite dimensional vector space admits a unique topology for which it becomes a topological vector space, we conclude that the Hermitian topology coincides with the symplectic weak topology on $\mathbf{S}$ when $\operatorname{dim}(\mathrm{S})<+\infty$.

In another situation, assume that $\mathrm{S}$ has a Hilbert structure

$$
\mathrm{S}=\mathrm{H}_{-} \oplus \mathrm{H}_{+} \text {with }\left[\mathrm{H}_{-}: \mathrm{H}_{+}\right]=0,
$$

say with a collection of Hilbert pairs, as typified by $\left\{\mathrm{H}_{ \pm}\right\}$. Then $\mathrm{S}$ itself can be considered as the direct sum Hilbert space (see Example 1.9] in Section 11) with a Hilbert norm $\|\cdot\|_{S}$, depending on the Hilbert pair $\left\{\mathrm{H}_{ \pm}\right\}$. Now consider another Hilbert pair $\left\{\hat{H}_{ \pm}\right\}$of the Hilbert structure for $\mathrm{S}$, so

$$
\mathrm{S}=\hat{\mathrm{H}}_{-} \oplus \hat{\mathrm{H}}_{+} \text {with }\left[\hat{\mathrm{H}}_{-}: \hat{\mathrm{H}}_{+}\right]=0 \text {, }
$$

and $S$ now has the corresponding Hilbert norm $\|\cdot\|_{\hat{S}}$. Then, as proved in [14, Section 4, Lemma 4.1] these two norms on $\mathrm{S}$ are equivalent, and so they define the same topology, called the Hilbert topology on S.

Since the Hilbert topology on $\mathbf{S}$ arises from a norm, $\mathrm{S}$ is then a locally convex topological vector space, in terms of its Hilbert topology. Furthermore, the Hilbert structure on $\mathrm{S}$ specifies a unique uniformity, as generated by the set of all open balls of all Hilbert norms (see [22, Chapter 6, page 178]), and this uniform topology coincides with the Hilbert topology.

In [14, Section 3] there is a comparison of interesting results for the Hilbert topology and the symplectic weak topology on S, as follows:

Remark 3.2. The following properties hold:

(1) The symplectic weak topology is weaker (i.e. not stronger, finer, larger) than the Hilbert topology on S. Moreover it is strictly weaker than the Hilbert topology if and only if $\mathbf{S}$ is infinite dimensional (in fact, in this case each open ball of a Hilbert norm is not weak-open on S). 
(2) A linear manifold in S is weak closed if and only if it is closed in the Hilbert topology - and hence a closed Hilbert subspace under each Hilbert norm on $\mathrm{S}$.

(3) The symplectic weak topology is metrizable if and only if $\mathrm{S}$ is finite dimensional; see [14, Section 3, Corollary 3.3].

\section{Finite dimensional Complex symplectic spaces}

4.1. Theory of separation and coupling for Lagrangian spaces. In this penultimate section of our survey and review paper we describe in more detail the boundary value theory for Lagrange symmetric ordinary differential operators on an arbitrary real interval $\mathfrak{I}=(a, b)$, for $-\infty \leq a<b \leq+\infty$, thereby involving singular as well as regular boundary value problems. We expand our description of the second-order Sturm-Liouville operator, as presented in Section 2.1, while emphasizing the novel advantages arising from the algebraic methods of complex symplectic spaces and their algebra.

Our earlier discussion of the Sturm-Liouville differential expression on an arbitrary interval $(a, b) \subset \mathbb{R}$ described the theory of self-adjoint operators so generated in the Hilbert function space $L^{2}((a, b) ; w)$ (for weight $w$ as described in Section 2.1. in particular (2.1) and (2.8)) in terms of the boundary complex symplectic space S; see (2.18). Indeed we indicated briefly how this boundary value theory could also apply to a Lagrange symmetric differential expression $M$ of higher order $n \geq 2$. Then the GKN-EM Theorem 1.14 asserts that each such self-adjoint operator $T$, generated by $M$ in $L^{2}((a, b) ; w)$, is defined by a complete Lagrangian $\mathrm{L} \subset \mathrm{S}$ (say $\operatorname{dim}(\mathrm{S})=2 d \leq 2 n$ and $\operatorname{Ex}(\mathrm{S})=0$ ) or basis $\left\{\mathrm{w}_{1}, \ldots, \mathrm{w}_{d}\right\}$ of vectors in $\mathrm{L}$, or equally well by a GKN-set of boundary functions (see [27, Chapter V, Section 18.1, Theorem 4]) $\left\{w_{1}, \ldots, w_{d}\right\}$ representing these basis vectors and thus specifying a symmetric set of boundary conditions.

In Section 4.2 we continue our investigations of this boundary value theory, but now with emphasis on the symplectically orthogonal decomposition

$$
\mathrm{S}=\mathrm{S}_{-} \oplus \mathrm{S}_{+},
$$

where $\mathrm{S}_{-}$and $\mathrm{S}_{+}$are the left and right boundary spaces, respectively, corresponding to the left and right endpoints of $\mathfrak{I}$, as given in Definition 4.7. With these methods we can make a precise analysis of the separation and coupling of the GKN-sets of boundary conditions and use these techniques to construct an intrinsic classification of the various kinds of complete Lagrangians $\mathrm{L}$ (and thereby of self-adjoint operators $T$ in $\left.L^{2}((a, b) ; w)\right)$ of higher order differential expressions.

In this exposition we first concentrate, in Section 4.1, on the abstract algebra of finite dimensional symplectic spaces and their Lagrangian subspaces, here based on the important Balanced Intersection Principle; see [10, Section III.1, Theorem 3]. After this study we turn in Section 4.2 to explicit applications for regular and singular boundary value problems for second and higher order linear ordinary differential equations.

Let $\mathrm{S}$ be a finite dimensional complex symplectic space with symplectic product $[\cdot: \cdot]$, as in Definition 1.1] and assume there exists a symplectically orthogonal direct sum decomposition (at this stage with no reference to any endpoints of intervals):

$$
\mathrm{S}=\mathrm{S}_{-} \oplus \mathrm{S}_{+} \text {with }\left[\mathrm{S}_{-}: \mathrm{S}_{+}\right]=0,
$$


as in Definition 1.5 of Section 11. Then the symplectic invariants of S are defined in (1.22) to (1.24) of Section 1 and are denoted as follows:

Remark 4.1. The complete set of symplectic invariants of $\mathbf{S}$ is $\{\mathbf{p}, \mathbf{q}\}$, where

(1) $\mathbf{p}$ is the positivity index, $\mathbf{q}$ is the negativity index, and further,

(2) $\Delta=\min \{\mathbf{p}, \mathbf{q}\}$ is the Lagrangian index, Dimension or $\operatorname{dim}(\mathrm{S}) \equiv D=\mathbf{p}+\mathbf{q}$, and Excess or $\operatorname{Ex}(\mathrm{S})=\mathbf{p}-\mathbf{q}$.

We denote the corresponding invariants of the symplectic spaces $\mathbf{S}_{-}$and $\mathbf{S}_{+}$, respectively, by

$$
\mathbf{p}_{ \pm}, \mathbf{q}_{ \pm}, \Delta_{ \pm}, D_{ \pm}, E x_{ \pm} .
$$

It is straightforward to conclude that

$$
\mathbf{p}=\mathbf{p}_{+}+\mathbf{p}_{-}, \quad \mathbf{q}=\mathbf{q}_{+}+\mathbf{q}_{-}, \quad \Delta \geq \Delta_{-}+\Delta_{+}
$$

and also

$$
D=D_{-}+D_{+} \text {and } \operatorname{Ex}(\mathrm{S})=E x\left(\mathrm{~S}_{-}\right)+E x\left(\mathrm{~S}_{+}\right) .
$$
if

Recall (see Section 3) that there exists a complete Lagrangian $\mathrm{L} \subset \mathrm{S}$ if and only

$$
E x(\mathrm{~S})=0\left(\text { or } \mathbf{p}=\mathbf{q}, \text { or } E x\left(\mathrm{~S}_{-}\right)=-E x\left(\mathrm{~S}_{+}\right)\right),
$$

and in this case (abbreviating $E x\left(\mathrm{~S}_{ \pm}\right)=E x_{ \pm}$, respectively)

$$
\Delta=\Delta_{-}+\Delta_{+}+\left|E x_{ \pm}\right|\left(\text {where }\left|E x_{ \pm}\right|=\left|E x_{+}\right|=\left|E x_{-}\right|\right) .
$$

Moreover, a Lagrangian $\mathrm{L} \subset \mathrm{S}$ is complete if and only if

$$
2 \operatorname{dim}(\mathrm{L})=\operatorname{dim}(\mathrm{S}) \text {, }
$$

so $\operatorname{dim}(\mathrm{S})$ is necessarily even in this case.

With these notations established, we next state the Balanced Intersection Principle (for the proof of this result see [10, Section III.1, Theorem 3]):

Theorem 4.2. Let $\mathrm{S}$ be a finite dimensional complex symplectic space, with symplectic product $[\cdot: \cdot]$, and a symplectically orthogonal direct sum decomposition, as in (4.1),

$$
\mathrm{S}=\mathrm{S}_{-} \oplus \mathrm{S}_{+} \text {with }\left[\mathrm{S}_{-}: \mathrm{S}_{+}\right]=0 .
$$

Assume $\operatorname{Ex}(\mathrm{S})=0$, and let $\mathrm{L}$ be a complete Lagrangian of $\mathrm{S}$.

Given this situation the Balanced Intersection Principle holds:

$$
0 \leq \Delta_{-}-\operatorname{dim}\left(\mathrm{L} \cap \mathrm{S}_{-}\right)=\Delta_{+}-\operatorname{dim}\left(\mathrm{L} \cap \mathrm{S}_{+}\right) \leq \min \left\{\Delta_{-}, \Delta_{+}\right\} .
$$

As we shall see later, the regular boundary value problem rests on the special case where

$$
\operatorname{dim}\left(\mathrm{S}_{-}\right)=\operatorname{dim}\left(\mathrm{S}_{+}\right), \text {equivalently } \Delta_{-}=\Delta_{+},
$$

as asserted in the next corollary:

Corollary 4.3. Let $\mathrm{S}$, with $[\cdot: \cdot]$, be a complex symplectic space of finite dimension, having the decomposition

$$
\mathrm{S}=\mathrm{S}_{-} \oplus \mathrm{S}_{+} \text {with }\left[\mathrm{S}_{-}: \mathrm{S}_{+}\right]=0,
$$

as in Theorem 4.2. Let $\mathrm{L} \subset \mathrm{S}$ be a complete Lagrangian, and assume further

$$
\operatorname{dim}\left(\mathrm{S}_{-}\right)=\operatorname{dim}\left(\mathrm{S}_{+}\right) \text {, equivalently } \Delta_{-}=\Delta_{+} .
$$


Then

$$
\operatorname{dim}\left(\mathbf{L} \cap \mathrm{S}_{-}\right)=\operatorname{dim}\left(\mathbf{L} \cap \mathrm{S}_{+}\right) .
$$

In anticipation of applications concerning the separation and coupling of boundary conditions, as described in Section 4.2, we next define these basic concepts within the abstract algebraic framework.

Definition 4.4. Let $\mathrm{S}$, with symplectic product $[\cdot: \cdot]$, be a finite dimensional complex symplectic space having a symplectically orthogonal decomposition

$$
\mathrm{S}=\mathrm{S}_{-} \oplus \mathrm{S}_{+} \text {with }\left[\mathrm{S}_{-}: \mathrm{S}_{+}\right]=0,
$$

as in (4.1) to (4.7).

Then a non-zero vector $v \in \mathrm{S}$ is:

(i) separated at the left in case $v \in \mathrm{S}_{-}$

(ii) separated at the right in case $v \in \mathrm{S}_{+}$

(iii) coupled otherwise, i.e. $\vee \notin \mathrm{S}_{-} \cup \mathrm{S}_{+}$(note: $\mathrm{S}_{-} \cup \mathrm{S}_{+}$is the set union, not the product of vector spaces).

Let $\mathrm{S}$, with symplectic product $[\cdot: \cdot]$, be a finite dimensional complex symplectic space having a symplectically orthogonal direct sum

$$
\mathrm{S}=\mathrm{S}_{-} \oplus \mathrm{S}_{+} \text {with }\left[\mathrm{S}_{-}: \mathrm{S}_{+}\right]=0,
$$

as in Definition 4.4. Assume $E x(\mathrm{~S})=0$ and let $\mathrm{L} \subset \mathrm{S}$ be a complete Lagrangian space, so $\operatorname{dim}(\mathrm{L})=\Delta$. Then a basis of vectors for $\mathrm{L}$ may contain vectors each of which is coupled, and other vectors, each of which is separated. We expand on these concepts in the next definition; however, for full details on these topics see [10, Section III.1].

Definition 4.5. Consider the finite dimensional complex symplectic space $\mathrm{S}$, with $\operatorname{Ex}(\mathrm{S})=0$, and assume

$$
\mathrm{S}=\mathrm{S}_{-} \oplus \mathrm{S}_{+} \text {with }\left[\mathrm{S}_{-}: \mathrm{S}_{+}\right]=0,
$$

as in (4.12); let $\mathrm{L} \subset \mathrm{S}$ be a complete Lagrangian subspace. A basis for $\mathrm{L}$ is called minimally coupled in case no other basis of $L$ has fewer coupled vectors. For each such minimally coupled basis for $\mathrm{L}$, the number of coupled vectors is called the necessary coupling of $L$ and is denoted by

$$
\operatorname{Nec} \text {-coupling }(\mathrm{L}):=\Delta-\operatorname{dim}\left(\mathrm{L} \cap \mathrm{S}_{-}\right)-\operatorname{dim}\left(\mathrm{L} \cap \mathrm{S}_{+}\right) \text {, }
$$

which is an integer in the range $[0, \Delta]$.

Particularly, define the concepts (compare [10, Section III.1]): L is strictly separated in case

$$
\text { Nec-coupling }(\mathrm{L})=0
$$

(i.e. there exists a basis for $\mathrm{L}$ with no coupled vectors), and $\mathrm{L}$ is totally coupled in case

$$
\text { Nec-coupling }(\mathrm{L})=\Delta
$$

(i.e. every basis for $\mathrm{L}$ consists entirely of coupled vectors).

In addition, an important parameter for complete Lagrangians $L$ of $S$ is the coupling grade of L, namely (see 4.9)

$$
\operatorname{grade}(\mathrm{L}):=\Delta_{-}-\operatorname{dim}\left(\mathrm{L} \cap \mathrm{S}_{-}\right)=\Delta_{+}-\operatorname{dim}\left(\mathrm{L} \cap \mathrm{S}_{+}\right) .
$$


A direct calculation verifies the identity (see [10, Section III.1, Corollary 3]),

$$
\text { Nec-coupling }(\mathrm{L})=2 \operatorname{grade}(\mathrm{L})+\left|E x_{ \pm}\right| \text {. }
$$

Further, in the references mentioned above, we find the major result:

$\{$ each complete Lagrangian $\mathrm{L} \subset \mathrm{S}$ has a minimally coupled basis, and each

\{ minimally coupled basis contains precisely the following vector numbers:

$\begin{cases}(\text { i }) & \operatorname{dim}\left(\mathrm{L} \cap \mathrm{S}_{-}\right)=\Delta_{-}-\text {grade }(\mathrm{L}) \text { vectors, each separated at the left; } \\ \text { (ii) } & \operatorname{dim}\left(\mathrm{L} \cap \mathrm{S}_{+}\right)=\Delta_{+}-\text {grade }(\mathrm{L}) \text { vectors, each separated at the right; and } \\ \text { (iii) } & \text { Nec-coupling }(\mathrm{L}) \text { vectors, each coupled. }\end{cases}$

The existence of a coupled Lagrangian $\mathrm{L} \subset \mathrm{S}$, with a prescribed grade $(\mathrm{L})=l$ (say), is guaranteed by the result given in [10, Section III.1, Theorem 4], namely:

$$
\left\{\begin{array}{l}
\text { For each non-negative integer } l \leq \min \left\{\Delta_{-}, \Delta_{+}\right\} \\
\text {there exists a complete Lagrangian } \mathrm{L}_{l} \subset \mathrm{S} \text { such that: }
\end{array}\right.
$$

and hence, by (4.18),

$$
\operatorname{Nec} \text {-coupling }\left(\mathrm{L}_{l}\right)=2 \operatorname{grade}\left(\mathrm{L}_{l}\right)+\left|E x_{ \pm}\right| .
$$

We are now in a position to illustrate, in Section 4.2, just how these results given in (4.1) to (4.23) of abstract symplectic algebra can apply to the classification of symmetric boundary conditions, specifying boundary value problems for Lagrange symmetric ordinary differential expressions $M$ on a real interval $\mathfrak{I}$; see (2.24) and (2.25) of Section 2.1 Full details for regular and singular problems of higher order differential expressions, with tabulations of important results, are presented in the monograph [10, Section III.2].

4.2. Applications for ordinary symmetric boundary conditions. In this section we record the generalization of the application of symplectic algebra, as introduced for the Sturm-Liouville differential expression in Section 2.1, to Lagrange symmetric ordinary differential expressions of order $n \geq 2$, which are specified on an arbitrary open interval of the real line - for instance, as in the classical differential expressions of the form (4.28). However, we choose to present this boundary value theory in even greater generality using quasi-differential expressions, as given in (4.25) and (4.26); this theory allows for the minimal local-integrability conditions on the complex-valued coefficients of the differential expression. We do not explicitly review or define the concepts for quasi-derivatives (i.e. certain linear combinations of classical derivatives, as prescribed for the coefficient entries of a Shin-Zettl matrix $A$; see below), but instead proceed on the basis of the thorough exposition of quasi-differential expressions, as given in the monograph [10, Section II].

The most general linear ordinary differential expressions so far defined, for order $n \in \mathbb{N}$ and $n \geq 2$, are the Shin-Zettl quasi-differential expressions; for details, in order of date of publication, see [29, [27, [35, 77, 8], [17, 9] and [10, Appendix A]. Quasi-differential expressions of order 1 need to be defined separately (see 9 , Section 3]) but are not considered here. 
Let $(a, b)$ be an arbitrary open interval of the real line $\mathbb{R}$. For $n \geq 2$ let $A=$ $\left[a_{r, s}\right] \in Z_{n}(a, b)$ be a Shin-Zettl complex-valued matrix of order $n$ and let $M_{A}$ be the corresponding quasi-differential expression generated by $A$. Here the elements $\left\{a_{r, s}: r, s=1,2, \ldots, n\right\}$ satisfy $a_{r, s}:(a, b) \rightarrow \mathbb{C}$ and are Lebesgue measurable.

Remark 4.6. We assume that the matrix $A$ is Lagrange symmetric (see [8, Section 10] and [17, Section 2]); it is this condition that ensures symmetry in the quasidifferential expression. This assumption is a generalization of the property of formal self-adjointness for classical linear ordinary differential expressions; see (4.28).

We denote by $\left\{f_{A}^{[r]}:(a, b) \rightarrow \mathbb{C}: r=0,1,2, \ldots, n\right\}$ the quasi-derivatives of $f$ with respect to $A$; for the definition see [8, Section 5], [17, Section 2] and [10, Section II]. These quasi-derivatives have the properties

$$
f_{A}^{[r]} \in A C_{\mathrm{loc}}(a, b) \text { for } r=0,1,2, \ldots, n-1 \text { and } f_{A}^{[n]} \in L_{\mathrm{loc}}^{1}(a, b) .
$$

The domain $D\left(M_{A}\right)$ of $M_{A}$ is defined by

$$
D\left(M_{A}\right):=\left\{f:(a, b) \rightarrow \mathbb{C}: f_{A}^{[r-1]} \in A C_{\mathrm{loc}}(a, b) \text { for } r=1,2, \ldots, n\right\}
$$

with

$$
M_{A}[f]:=i^{n} f_{A}^{[n]} \text { for all } f \in D\left(M_{A}\right),
$$

where $i$ is the complex unit $i^{2}=-1$; thus $M_{A}[f] \in L_{\text {loc }}^{1}(a, b)$.

The minimal conditions on the elements of the matrix $A$, in order to define the quasi-derivatives, are

$$
\left\{a_{r, s}(\cdot) \in L_{\mathrm{loc}}^{1}(a, b): r, s=1,2, \ldots, n\right\} ;
$$

if these elements are smooth, say $\left\{a_{r, s}(\cdot) \in C^{(n)}(a, b): r, s=1,2, \ldots, n\right\}$, then the quasi-derivatives reduce to linear combinations of classical derivatives for $f$ on $(a, b)$. In this case the differential expression takes on the classical form (4.28) (see [17. Section 2]), in comparison with the quasi-differential form (4.26),

$$
M_{A}[f]=\sum_{r=0}^{m}\left(p_{r} f^{(r)}\right)^{(r)}+i \sum_{r=0}^{n-m-1}\left[\left(q_{r} f^{(r)}\right)^{(r+1)}+\left(q_{r} f^{(r+1)}\right)^{(r)}\right] .
$$

Here, given the positive integer $n$, the real-valued functions $\left\{p_{r}\right\}$ and $\left\{q_{r}\right\}$ for $r=0,1,2, \ldots m$ are suitably smooth, and $m=[n / 2]$ is the integer part of $n / 2$. Thus all classical linear ordinary differential expressions are special cases of the general class of quasi-differential expressions; see [10, Appendix A].

The advantage of the use of quasi-derivatives, accepting the initial complication in their definition, is that the minimal conditions on the coefficients (4.27) are applicable, and the resulting form of Green's formula for the differential expression $M_{A}$ is shorter and more useful in applications; see (4.29), (4.30) and (4.31).

The Lagrange symmetric form of the matrix $A$ determines the symmetric form of Green's formula for the quasi-differential expression $M_{A}$; i.e. for all $f, g \in D\left(M_{A}\right)$

$$
\int_{\alpha}^{\beta}\left\{\bar{g}(x) M_{A}[f](x)-f(x) \overline{M_{A}[g]}(x)\right\} d x=[f, g]_{A}(\beta)-[f, g]_{A}(\alpha)
$$

for any compact interval $[\alpha, \beta] \subset(a, b)$. Here the symplectic (skew-symmetric sesquilinear) form

$$
[\cdot, \cdot]_{A}(x): D\left(M_{A}\right) \times D\left(M_{A}\right) \rightarrow \mathbb{C} \text { for all } x \in(a, b)
$$


has the explicit representation

$$
[f, g]_{A}(x)=i^{n} \sum_{r=1}^{n}(-1)^{r-1} f_{A}^{[n-r]}(x) \overline{g_{A}^{[r-1]}}(x) .
$$

The Sturm-Liouville differential expression $M$ as considered in Section 2.1 (see (2.3) and (2.4) under the minimal conditions (2.1)) is an example of a quasidifferential expression for which the Shin-Zettl matrix is

$$
A=\left[\begin{array}{cc}
0 & p^{-1} \\
q & 0
\end{array}\right]
$$

the quasi-derivatives in this case are, where the prime ' denotes classical differentiation:

$$
f_{A}^{[0]}=f, f_{A}^{[1]}=p f^{\prime} \text { and } f_{A}^{[2]}=\left(p f^{\prime}\right)^{\prime}-q f .
$$

The general Green formula (4.29) then reduces to the Sturm-Liouville case as given in (2.6) and (2.7).

In this general case the analysis of boundary value problems for the differential expression $M_{A}$ given in (4.26) follows the analysis in Section 2.1. Given the weight function $w$, as in (2.1), the analysis takes place in the weighted Hilbert function space $L^{2}((a, b) ; w)$ with norm and scalar product given by

$$
\|f\|_{w}^{2}=\int_{a}^{b} w(x)|f(x)|^{2} d x \text { and }(f, g)_{w}=\int_{a}^{b} w(x) f(x) \bar{g}(x) d x .
$$

As in Section 2.1 the maximal operator $T_{1}$ generated by the expression $M_{A}$ in this space is defined by

$$
D\left(T_{1}\right):=\left\{f \in D\left(M_{A}\right): f, w^{-1} M_{A}[f] \in L^{2}((a, b) ; w)\right\}
$$

and

$$
T_{1} f:=w^{-1} M_{A}[f] \text { for all } f \in D\left(T_{1}\right) .
$$

From Green's formula (4.29) it follows that the two limits

$$
[f, g]_{A}(b):=\lim _{x \rightarrow b}[f, g]_{A}(x) \text { and }[f, g]_{A}(a):=\lim _{x \rightarrow a}[f, g]_{A}(x)
$$

both exist and are finite in $\mathbb{C}$, for all $f, g \in D\left(T_{1}\right)$.

The minimal operator $T_{0}$ generated by the expression $M_{A}$ in this space is defined by the dense domain

$$
D\left(T_{0}\right):=\left\{f \in D\left(T_{1}\right): \lim _{x \rightarrow b}[f, g]_{A}(x)-\lim _{x \rightarrow a}[f, g]_{A}(x)=0 \text { for all } g \in D\left(T_{1}\right)\right\}
$$

and

$$
T_{0} f:=w^{-1} M_{A}[f] \text { for all } f \in D\left(T_{0}\right) .
$$

From established results in [27, Chapter V], 9] and [10, Appendix] it is known that the following properties of $T_{0}$ and $T_{1}$ hold, where * denotes an adjoint operator in $L^{2}((a, b) ; w)$ :

$$
\begin{cases}(\text { i }) & D\left(T_{0}\right) \subseteq D\left(T_{1}\right) \text { and } T_{0} \subseteq T_{1} \\ \text { (ii }) & T_{0} \text { is a closed, symmetric operator in } L^{2}((a, b) ; w) \\ \text { (iii) } & T_{1} \text { is a closed operator in } L^{2}((a, b) ; w) \\ \text { (iv) } & T_{0}^{*}=T_{1} \text { and } T_{1}^{*}=T_{0} .\end{cases}
$$


The deficiency indices $\left\{d^{-}, d^{+}\right\}$of the operator $T_{0}$ (see Example 1.13) satisfy the inequalities

$$
0 \leq d^{-}, d^{+} \leq n
$$

where $n$ is the order of the quasi-differential expression $M_{A}$. In general, since the elements of the Shin-Zettl matrix $A$ may be complex-valued on $(a, b)$, the indices $\left\{d^{-}, d^{+}\right\}$may be unequal. As mentioned in Example 1.13 there are self-adjoint extensions to the closed symmetric operator $T_{0}$ if and only if these indices are equal. Thus we make the additional condition on the matrix $A$ that (see (1.48) $)$

$$
d^{-}=d^{+} .
$$

There are no known necessary and sufficient conditions on the matrix $A$ to determine the values of the non-negative integers $\left\{d^{ \pm}\right\}$and so to decide if condition (4.38) is satisfied. There is substantial literature devoted to sufficient conditions on the elements of the matrix $A$ to determine the indices $\left\{d^{ \pm}\right\}$; see [27, Chapter VI] and 7 .

The (degenerate) symplectic product $[\cdot, \cdot]: D\left(T_{1}\right) \times D\left(T_{1}\right) \rightarrow \mathbb{C}$ is defined by

$$
[f, g]:=\left(T_{1} f, g\right)_{w}-\left(f, T_{1} g\right)_{w} \text { for all } f, g \in D\left(T_{1}\right) .
$$

From Green's formula (4.29), definition (4.33) of the operator $T_{1}$, and the limit result (4.34) it follows that

$$
[f, g]=[f, g]_{A}(b)-[f, g]_{A}(a) \text { for all } f, g \in D\left(T_{1}\right) .
$$

The boundary complex symplectic space generated by the quasi-differential expression $M_{A}$ is defined as in Section 2.1 (see (2.18) and (2.20) ) and also in 11, Appendix], namely (recall Notation 1.3)

$$
\mathrm{S}:=D\left(T_{1}\right) / D\left(T_{0}\right)
$$

with the (non-degenerate) symplectic product

$$
[\mathrm{f}: \mathrm{g}]:=[f, g]=[f, g]_{A}(b)-[f, g]_{A}(a) \text { for all } \mathrm{f}, \mathrm{g} \in \mathrm{S}
$$

for cosets $\mathrm{f}=\left\{f+D\left(T_{0}\right)\right\}, \mathrm{g}=\left\{g+D\left(T_{0}\right)\right\}$. Here $f, g \in D\left(T_{1}\right)$ are corresponding representative functions for these cosets $f$ and $g$.

From the deficiency index conditions (4.37) and (4.38), and defining $d:=d^{-}=$ $d^{+}$, we obtain

$$
0 \leq \operatorname{dim}(\mathrm{S})=d^{-}+d^{+}=2 d \leq 2 n \text { and } \operatorname{Ex}(\mathrm{S})=0 .
$$

Next we define the left and right boundary spaces $\mathbf{S}_{-}$and $\mathbf{S}_{+}$, respectively, for this boundary complex symplectic space $\mathbf{S}$, corresponding to the endpoints $a<b$ of the interval $(a, b)$.

Definition 4.7. Consider the boundary complex symplectic space $\mathrm{S}$, and the symplectic product $[\cdot: \cdot]$, for the quasi-differential expression $M_{A}$ on the interval $(a, b)$, as in (4.41) and (4.42), say

$$
\mathrm{S}:=D\left(T_{1}\right) / D\left(T_{0}\right)
$$

and consider the natural projection map (see (1.52)

$$
\Psi: D\left(T_{1}\right) \rightarrow \mathrm{S} \text { given by } f \rightarrow \mathrm{f}=\left\{f+D\left(T_{0}\right)\right\} .
$$

Also consider the subspaces of $D\left(T_{1}\right)$ :

$$
D_{-}\left(T_{1}\right):=\left\{f \in D\left(T_{1}\right): \text { some neighborhood of } b \text { does not intersect } \operatorname{supp}(f)\right\}
$$


$(4.46)$

$D_{+}\left(T_{1}\right):=\left\{f \in D\left(T_{1}\right):\right.$ some neighborhood of $a$ does not intersect $\left.\operatorname{supp}(f)\right\}$.

Then define the left and right boundary spaces, respectively,

$$
\mathrm{S}_{-}:=\Psi D\left(T_{-}\right) \text {and } \mathrm{S}_{+}:=\Psi D\left(T_{+}\right) .
$$

Remark 4.8. The existence of non-null elements in the subspaces $D_{ \pm}\left(T_{1}\right)$ depends on the use of the "Naimark patching lemma"; see 27, Chapter V, Section 17.3, Lemma 2].

It is clear that $S_{ \pm}$are linear subspaces of $S$, and a non-trivial argument based on the Naimark patching lemma (see [10, Section III.2, Theorem 5]) proves that $\mathrm{S}=\operatorname{span}\left\{\mathrm{S}_{-}, \mathrm{S}_{+}\right\}$, in fact the following theorem:

Theorem 4.9. Consider the boundary complex symplectic space $\mathrm{S}$, with symplectic product $[\cdot: \cdot]$, generated by $M_{A}$ on $(a, b)$, as given in Definition 4.7. Then the left and right boundary spaces $\mathbf{S}_{-}$and $\mathrm{S}_{+}$, respectively, provide a symplectically orthogonal decomposition

$$
\mathrm{S}=\mathrm{S}_{-} \oplus \mathrm{S}_{+} \text {with }\left[\mathrm{S}_{-}: \mathrm{S}_{+}\right]=0 .
$$

Thus both $\mathrm{S}_{-}$and $\mathrm{S}_{+}$are symplectic subspaces of $\mathrm{S}_{\text {. }}$

We now seek to refine the general theory of self-adjoint operators, say $T$ on $D(T)$, generated by the quasi-differential expression $M_{A}$ in the Hilbert function space $L^{2}((a, b) ; w)$; see (4.36). Such operators $T$ exist (see the deficiency condition (4.38) ) and satisfy

$$
D\left(T_{0}\right) \subseteq D(T) \subseteq D\left(T_{1}\right) \text { and } T_{0} \subseteq T=T^{*} \subseteq T_{1} .
$$

In particular we seek to incorporate information about the left and right boundary spaces $\mathrm{S}_{-}$and $\mathrm{S}_{+}$, and so to investigate the concepts of separation and coupling of boundary conditions at the endpoints $a$ and $b$ of the interval $(a, b)$.

According to the GKN-EM Theorem 1.14 of Section1, we can define and describe any such operator $T$ and domain $D(T)$ as follows:

$$
D(T)=\left\{f \in D\left(T_{1}\right):[\mathrm{f}: \mathrm{L}]=0\right\}
$$

$$
T f=T_{1} f \text { for all } f \in D(T),
$$

where $\mathrm{L} \subset \mathrm{S}$ is any complete Lagrangian subspace of S. Recalling (see (4.43)) that $\operatorname{dim}(\mathrm{S})=2 d$ with $d \in \mathbb{N}$ and that (see (3.9) $) 2 \operatorname{dim}(\mathrm{L})=\operatorname{dim}(\mathrm{S})$, one sees that $\operatorname{dim}(\mathrm{L})=d$. Thus if we select any basis of $\mathrm{L}$, say $\left\{\mathrm{w}_{1}, \mathrm{w}_{2}, \ldots, \mathrm{w}_{d}\right\}$, noting that, from the definition of a Laplacian subspace, Definition [1.7, the symmetry conditions

$$
\left[\mathrm{w}_{r}: \mathrm{w}_{s}\right]=0 \text { for all } r, s=1,2, \cdots, d
$$

are satisfied, then we can write

$$
D(T)=\left\{f \in D\left(T_{1}\right):\left[\mathrm{f}: \mathrm{w}_{r}\right]=0 \text { for } r=1,2, \ldots, d\right\} .
$$

In Section 2.1. say (2.23) to (2.27) and the related discussion, we define a boundary condition by a function $w \in D\left(T_{1}\right)$. Namely we define the corresponding functional $F_{w}$ by (compare (3.13) of Section 3)

$$
F_{w}: D\left(T_{1}\right) \rightarrow \mathbb{C} \text { as given by } f \rightarrow[f, w] .
$$


Then the generalised boundary condition is the null space of the functional $F_{w}$ in $D\left(T_{1}\right)$; compare (4.54) and introduce the abbreviation, i.e.

$$
[f, w]=0 \text { for } f \in D\left(T_{1}\right)
$$

or, equally well, see (4.40),

$$
[f, w]_{A}(b)-[f, w]_{A}(a)=0 .
$$

Definition 4.10. Consider a boundary condition function $w \in D\left(T_{1}\right)$ satisfying $w \notin D\left(T_{0}\right)$.

Then we say that (compare Definition 4.4):

(i) $w$ is left separated in case $\mathbf{w}=\Psi w \in \mathbf{S}_{-}$

(ii) $w$ is right separated in case $\mathbf{w}=\Psi w \in \mathbf{S}_{+}$

(iii) $w$ is coupled (at endpoints of $(a, b))$ in case $\mathbf{w} \notin \mathbf{S}_{-} \cup \mathbf{S}_{+}$.

Lemma 4.11. The following three assertions are logically equivalent:

(i) $w \in D\left(T_{1}\right)$ is left separated (so $\mathbf{w} \in \mathrm{S}_{-}$is left separated).

(ii) $w \in D_{-}\left(T_{1}\right)\left(\bmod D\left(T_{0}\right)\right)$; i.e. there exists $\hat{w} \in D_{-}\left(T_{1}\right)$ such that $w-\hat{w} \in$ $D\left(T_{0}\right)$.

(iii) $\left[D\left(T_{1}\right), w\right]_{A}(b)=0$ so that $\left[D\left(T_{1}\right), w\right]=-\left[D\left(T_{1}\right), w\right]_{A}(a)$.

Remark 4.12. Following Lemma 4.11 we have

(i) Similar results to $(i),(i i)$ and $(i i i)$ hold for $w \in D_{+}\left(T_{1}\right)\left(\bmod D\left(T_{0}\right)\right)$ if $w$ is right separated (or $w \in \mathrm{S}_{+}$).

(ii) Further $w \in D\left(T_{1}\right)$ is coupled (or $\mathbf{w} \notin \mathrm{S}_{-} \cup \mathrm{S}_{+}$) if and only if $u \in \mathrm{w}$ (and $\left.u \notin D\left(T_{0}\right)\right)$ implies that $\operatorname{supp}(u)$ intersects every neighborhood of $a$, also of $b$; equivalently if and only if

$$
\left[D\left(T_{1}\right), w\right]_{A}(a) \neq 0 \text { and }\left[D\left(T_{1}\right), w\right]_{A}(b) \neq 0 .
$$

From these concepts and remarks we can reformulate our previous discussions on complete Lagrangians and their minimally coupled bases (see Definition 4.5 and also (4.17), (4.18) and 4.19) ) in terms of boundary conditions.

Extending the methods of the Sturm-Liouville theory in (2.23), (2.24) and (2.25) of Section 2.1, we note that a GKN symmetric set of boundary conditions for the general case (see [14, Section 5, Propositions 5.1 and 5.2]) consists of a set of functions in $D\left(T_{1}\right)$,

$$
\left\{w_{1}, w_{2}, \ldots, w_{d}\right\}
$$

such that their image under the natural projection map $\Psi$ (see (1.52)),

$$
\mathrm{w}_{r}=\Psi w_{r}=\left\{w_{r}+D\left(T_{0}\right)\right\} \text { for } r=1,2, \ldots, d,
$$

constitutes a basis $\left\{w_{1}, w_{2}, \ldots, w_{d}\right\}$ for a Lagrangian subspace $L$ in $S$; $L$ is necessarily complete since $\operatorname{dim}(\mathrm{S})=2 \operatorname{dim}(\mathrm{L})=2 d$. Note that since $\mathrm{L}$ is a Lagrangian subspace

$$
\left[\mathrm{w}_{r}: \mathrm{w}_{s}\right]=0 \text { for } r, s=1,2, \ldots, d,
$$

which implies that

$$
\left[w_{r}, w_{s}\right]=0 \text { for } r, s=1,2, \ldots, d ;
$$

this is the original GKN condition for the boundary condition set (4.57) as given in [27, Chapter V, Section 18.1, Theorem 4] and [9, Section 2]. 
The domain $D(T)$ of the self-adjoint operator $T$ generated by the set 4.57) (see also (4.49) $)$ is

$$
D(T)=\left\{f \in D\left(T_{1}\right):\left[f, w_{r}\right]=0 \text { for } r=1,2, \ldots, d\right\},
$$

or, equally well, using the notation (4.56)

$$
\left[f, w_{r}\right]_{A}(b)-\left[f, w_{r}\right]_{A}(a)=0 \text { for } r=1,2, \ldots, d .
$$

Theorem 4.13. Let the boundary complex symplectic space, of the quasi-differential expression $M_{A}$ on the interval $(a, b)$, be

$$
\mathrm{S}=D\left(T_{1}\right) / D\left(T_{0}\right)=\mathrm{S}_{-} \oplus \mathrm{S}_{+},
$$

with symplectic product $[\cdot: \cdot]$, as defined in Theorem 4.9.

Consider a GKN symmetric set of boundary condition functions

$$
\left\{w_{1}, w_{2}, \ldots, w_{d}\right\} \text { in } D\left(T_{1}\right)
$$

(with the corresponding vectors $\left\{\mathrm{w}_{1}, \mathrm{w}_{2}, \ldots, \mathrm{w}_{d}\right\}$ in $\mathrm{S}$, providing a basis for a complete Lagrangian $\mathrm{L} \subset \mathrm{S}$ ).

Now denote by

$$
\left\{\begin{array}{l}
\alpha=\text { number of left separated functions amongst }\left\{w_{1}, w_{2}, \ldots, w_{d}\right\} \\
\beta=\text { number of right separated functions amongst }\left\{w_{1}, w_{2}, \ldots, w_{d}\right\} \\
\gamma=\text { number of coupled functions amongst }\left\{w_{1}, w_{2}, \ldots, w_{d}\right\} .
\end{array}\right.
$$

Then conclude that (recall (4.14) for the definition of Nec-coupling(L))

$$
\left\{\begin{array}{l}
\alpha \leq \operatorname{dim}\left(\mathrm{L} \cap \mathrm{S}_{-}\right) \\
\beta \leq \operatorname{dim}\left(\mathrm{L} \cap \mathrm{S}_{+}\right) \\
\gamma \geq \operatorname{Nec} \text {-coupling }(\mathrm{L}) .
\end{array}\right.
$$

Further, if the GKN set $\left\{w_{1}, w_{2}, \ldots, w_{d}\right\}$ (or the basis $\left\{\mathrm{w}_{1}, \mathrm{w}_{2}, \ldots, \mathrm{w}_{d}\right\}$ ) is minimally coupled, that is

$$
\gamma=\operatorname{Nec}-\operatorname{coupling}(\mathrm{L})
$$

then

$$
\begin{aligned}
& \alpha=\operatorname{dim}\left(\mathrm{L} \cap \mathrm{S}_{-}\right) \\
& \beta=\operatorname{dim}\left(\mathrm{L} \cap \mathrm{S}_{+}\right) .
\end{aligned}
$$

Moreover, for each complete Lagrangian $\mathrm{L} \subset \mathrm{S}$, there exists a minimally coupled GKN symmetric set of boundary condition functions that defines a minimally coupled basis for $\mathrm{L}$.

Furthermore, for each triple of integers $\alpha, \beta$ and $\gamma$ with

$$
0 \leq \alpha \leq \operatorname{dim}\left(\mathrm{L} \cap \mathrm{S}_{-}\right) \quad 0 \leq \beta \leq \operatorname{dim}\left(\mathrm{L} \cap \mathrm{S}_{+}\right) \quad \gamma=\Delta-\alpha-\beta
$$

there exists a basis for $\mathrm{L}$ containing exactly

$\gamma$ vectors, each coupled $\alpha$ vectors, each separated at the left $\beta$ vectors, each separated at the right.

Proof. For a complete discussion of these results and for a proof of this theorem, see [11, Section 2, Corollary 2]. 
Example 4.14. Take $M_{A}$ on $(a, b)$, as above, of order $n=4$, and let the ShinZettl matrix $A$ have real-valued coefficients on $(a, b)$; thus $\operatorname{dim}(\mathrm{S})=2 d \leq 8$ and $E x(\mathrm{~S})=0$.

We consider the regular boundary value problem for $M_{A}$ on a compact interval $[a, b]$ with $-\infty<a<b<+\infty$. That is, we assume the minimal conditions (4.27) on the coefficients $\left\{a_{r s}: r, s=1,2,3,4\right\}$ are strengthened to require that $a_{r s} \in L^{1}(a, b)$ for $r, s=1,2,3,4$.

In this case it is known (see [10, Section IV]) that $d=4$ so $\operatorname{dim}(\mathrm{S})=8$, and also $\Delta_{-}=\Delta_{+}=2$ with $\left|E x_{ \pm}\right|=0$. Hence each complete Lagrangian $\mathrm{L} \subset \mathrm{S}$ has $\operatorname{dim}(\mathrm{L})=4$ and $\operatorname{grade}(\mathrm{L})=0,1$ or 2 .

For a simple case, suppose that grade $(\mathrm{L})=1$. Then

$$
\operatorname{Nec} \text {-coupling }(\mathrm{L})=2 \operatorname{grade}(\mathrm{L})=2 \text {; }
$$

therefore each minimally coupled basis of boundary condition functions contains exactly

$$
\begin{aligned}
& 2 \text { coupled boundary conditions } \\
& 1 \text { left separated boundary condition } \\
& 1 \text { right separated boundary condition. }
\end{aligned}
$$

All the three possible cases of grade $(\mathrm{L})=0,1$ or 2 are considered in [10, Example 1 , Section V, page 76] and are reported here as follows:

Interval: $[a, b]$, Order: $n=4$, Parameters: $d=\Delta=4$

(i) $\operatorname{grade}(\mathrm{L})=0$

Nec-coupling $(\mathrm{L})=0 \quad \operatorname{dim}\left(\mathrm{L} \cap \mathrm{S}_{ \pm}\right)=2$

2 left separated boundary conditions and 2 right separated boundary conditions

(ii) $\operatorname{grade}(\mathrm{L})=1$

Nec-coupling $(L)=2 \quad \operatorname{dim}\left(L \cap S_{ \pm}\right)=1$

1 left separated boundary condition and 1 right separated boundary condition

2 coupled boundary conditions

(iii) $\operatorname{grade}(\mathrm{L})=2$

Nec-coupling $(\mathrm{L})=4 \quad \operatorname{dim}\left(\mathrm{L} \cap \mathrm{S}_{ \pm}\right)=0$

4 coupled boundary conditions.

Example 4.15. Take $M_{A}$ on $[a, b)$ of order $n=4$; let the Shin-Zettl matrix $A$ have real-valued coefficients on $[a, b)$. We consider the boundary value problem for $M_{A}$ on an interval $[a, b)$ with $-\infty<a<b \leq+\infty$. That is, we assume the minimal conditions (4.27) on the coefficients $\left\{a_{r s}: r, s=1,2,3,4\right\}$ are strengthened to require that $a_{r s} \in L_{\text {loc }}^{1}[a, b)$ for $r, s=1,2,3,4$. Further assume that the weight $w \in L_{\text {loc }}^{1}[a, b)$. Then the boundary value problem is in general singular on $[a, b)$, but could be regular if $b \in \mathbb{R}$.

In this case it is known, when $E x(\mathrm{~S})=0$ (see [10, Section V, Example 3]), that $d=2,3$ or 4 , so $\operatorname{dim}(\mathrm{S})=4,6$ or 8 and also, respectively, $\Delta_{-}=\Delta_{+}=0,1$ or 2 , all with $\left|E x_{ \pm}\right|=0$. Hence each complete Lagrangian $\mathrm{L} \subset \mathrm{S}$ has $\operatorname{dim}(\mathrm{L})=2,3$ or 4 ; independently then grade $(\mathrm{L})=0,1$ or 2 . 
All the possible cases of $d=2,3$ or 4 and $\operatorname{grade}(\mathrm{L})=0,1$ or 2 are considered in [10. Section V, page 107, Example 3, order $n=4$ ] and are reported here (for minimally coupled bases) as follows:

(i) $d=2, \Delta_{-}=2, \Delta_{+}=0$ and $\operatorname{grade}(\mathrm{L})=0$

2 left separated boundary conditions and 0 right separated boundary conditions

0 coupled boundary conditions

(ii) $d=3, \Delta_{-}=2, \Delta_{+}=1$ and $\operatorname{grade}(\mathrm{L})=0$

2 left separated boundary conditions and 1 right separated boundary condition

0 coupled boundary conditions

(iii) $d=3, \Delta_{-}=2, \Delta_{+}=1$ and grade $(\mathrm{L})=1$

1 left separated boundary condition and 0 right separated boundary condition

2 coupled boundary conditions

(iv) $d=4, \Delta_{-}=2, \Delta_{+}=2$ and grade $(\mathrm{L})=0$

2 left separated boundary conditions and 2 right separated boundary conditions

0 coupled boundary conditions

(v) $d=4, \Delta_{-}=2, \Delta_{+}=2$ and $\operatorname{grade}(\mathrm{L})=1$

1 left separated boundary condition and 1 right separated boundary condition

2 coupled boundary conditions

(vi) $d=4, \Delta_{-}=2, \Delta_{+}=2$ and grade $(\mathrm{L})=2$

0 left separated boundary conditions and 0 right separated boundary conditions

4 coupled boundary conditions.

Example 4.16. Take $M_{A}$ on $[0 . \infty)$ of order $n=2$, let the real-valued Shin-Zettl matrix $A$ have the Sturm-Liouville form (see (4.32)

$$
A=\left[\begin{array}{cc}
0 & p^{-1} \\
q & 0
\end{array}\right],
$$

and follow the general results in 2.1

In this case we further assume that all three of $p, q, w \in C[0, \infty)$ and that $p(x)>0$ and $w(x)>0$ for all $x \in[0, \infty)$ to give 0 as a regular endpoint of the Sturm-Liouville differential equation (2.2), relative to this singular boundary value problem.

These conditions on $M_{A}$ and the weight $w$ imply that (see (2.22) of Section 2.1)

$$
\begin{gathered}
d^{-}=d^{+}=d=1 \text { or } 2 \\
\operatorname{dim}(\mathrm{S})=2 d \leq 2 n=4 \text { and } \operatorname{Ex}(\mathrm{S})=0 .
\end{gathered}
$$

Then following the results given in [10, Section IV, page 76, and Section V, page 107]:

(i) $d=1 \quad \Delta_{-}=1 \quad \Delta_{+}=0 \quad \operatorname{dim}(\mathrm{L})=1$

$\operatorname{grade}(\mathrm{L})=0$

1 left separated boundary condition and 0 right separated boundary condition

(ii) $d=2 \quad \Delta_{-}=1 \quad \Delta_{+}=1 \quad \operatorname{dim}(\mathrm{L})=2$

(a) $\operatorname{grade}(\mathrm{L})=0$ 
1 left separated boundary condition and 1 right separated boundary condition

0 coupled boundary conditions

(b) $\operatorname{grade}(\mathrm{L})=1$

0 left separated boundary condition and 0 right separated boundary condition

2 coupled boundary conditions.

\section{Conclusions: COMPARISONS, PRoblems, CONJECTURES}

The modern theory of boundary value problems for Lagrange symmetric (formally self-adjoint) linear differential expressions (formal operators) was initiated early in the previous century by the seminal discoveries of Weyl and Titchmarsh (see [32] and 33]), then incorporated into the general spectral theory of symmetric linear operators on appropriate Hilbert spaces by Stone and von Neumann (see [31]), and later amended by certain algebraic methods in diverse papers of Glazman, Krein and Naimark (see [2] and [27]). This development generated a vast and still growing literature of pure and applied mathematics (see the various treatises [2], 5], 6], 20], 27] and [30, especially their references and bibliographies).

The theory of complex symplectic spaces, as advanced by the current authors over the past dozen years (see [7] to [18), presents new viewpoints and insights into the unification and systemization of this theory of boundary value problems for ordinary differential, quasi-differential and partial differential operators; see Sections 2 and 4 Through a novel approach utilizing the symplectic algebra and the symplectic weak topology of the boundary complex symplectic space $\mathbf{S}$ of a given closed symmetric linear operator $T_{0}$ with dense domain $D\left(T_{0}\right)$ in a Hilbert space $H$, new results are found for ordinary and quasi-differential problems (regular and singular) - compare the Balanced Intersection Principle and the separationcoupling grades in Section 4.2 and in [10] and [11] - and also for elliptic partial differential problems - compare the Dirichlet and Harmonic Lagrangians and their corresponding self-adjoint operators in Section 2.2 and [13, [14, [15].

This universal and axiomatic overview of boundary value problems offers some simple definite answers to frequently vaguely formulated questions. For instance: What is a boundary condition? It is a vector $\mathbf{w} \in \mathbf{S}$ (or function $w \in D\left(T_{1}\right.$ ) with $\Psi w=\mathrm{w}$ ), interpreted as a linear functional on S (see Definition 1.12), and further a symmetric set of boundary conditions determining a self-adjoint operator $T$ on $D(T) \subset H$ is a complete Lagrangian $\mathrm{L} \subset \mathrm{S}$ (or a basis for $\mathrm{L}$ ), with $\Psi(D(T))=\mathrm{L}$ as in the GKN-EM Theorem 1.14. However, full appreciation of the global algebraic and topological structure of $\mathrm{S}$ leads to much deeper and unexplored aspects of boundary value theory - and we illustrate some of these new directions for research by several examples, unsolved questions and conjectures.

Example 5.1. As in Section 2.1, first examine the simplest Sturm-Liouville problem

$$
M[y]:=-y^{\prime \prime} \text { on }[0,1] \subset \mathbb{R},
$$

with boundary conditions, separated and coupled respectively:

$$
\text { Separated: } y(0)=y(1)=0 \quad \text { Coupled: } y(0)=y(1) \text { and } y^{\prime}(0)=y^{\prime}(1) \text {, }
$$


generating corresponding complete Lagrangians $\mathrm{L}_{\text {sep }}, \mathrm{L}_{\text {coup }} \subset \mathrm{S}$, and the two selfadjoint operators in the Hilbert space $L^{2}(0,1)$,

$$
T_{\text {sep }} \text { on } D\left(T_{\text {sep }}\right) \quad T_{\text {coup }} \text { on } D\left(T_{\text {coup }}\right) \text {. }
$$

Problem 5.2. Is there a continuous deformation of $T_{\text {sep }}$ into $T_{\text {coup }}$, with all intermediate stages corresponding to boundary conditions (of the same form as of (5.2) ) defining self-adjoint operators?

The answer appears to be affirmative from the fact (see [10, page 63]) that the set of all $d$-dimensional Lagrangian subspaces of $\mathbf{S} \approx \mathbb{C}^{2 d}$ (for integers $d \geq 1$ ) has a natural topology as a compact connected manifold, say

$$
\operatorname{Lag}_{\mathbb{C}}(d, 2 d) \approx U(d),
$$

i.e. homeomorphic to the group of all $d \times d$ unitary matrices. However the explicit display of the deformation is not readily available.

To present this problem in a more general format, consider the space $\operatorname{Lag}_{\mathbb{C}}(d, 2 d)$ as arising from a complex symplectic $\mathrm{S}$, as in Definition 4.5

$$
\mathrm{S}=\mathrm{S}_{-} \oplus \mathrm{S}_{+} \text {with }\left[\mathrm{S}_{-}: \mathrm{S}_{+}\right]=0,
$$

with $\operatorname{dim}(\mathrm{S})=2 d$ and $E x(\mathrm{~S})=0$. Here each Lagrangian subspace $\mathrm{L} \subset \mathrm{S}$ has a grade (see Definition 4.5) satisfying

$$
0 \leq \operatorname{grade}(\mathrm{L}) \leq \min \left\{\Delta_{-}, \Delta_{+}\right\} .
$$

Conjecture 5.3. For each integer $l$ satisfying $0 \leq l \leq \min \left\{\Delta_{-}, \Delta_{+}\right\}$the subset of $\operatorname{Lag}_{\mathbb{C}}(d, 2 d)$ defined by

$$
\operatorname{grade}(\mathrm{L})=l
$$

is connected.

Example 5.4. Consider again the Sturm-Liouville problem of Example [5.1, It is intuitively clear that the separated boundary conditions in (5.2) determine the subset $D\left(T_{\mathrm{sep}}\right) \subset D\left(T_{1}\right)$ by local behavior near the endpoint 0 and also near the endpoint 1 , whereas this statement is false for the coupled boundary conditions in (5.2) which are of a global nature (i.e. not locally determined) demanding simultaneous restrictions at both endpoints together. The concept of local - versus - global boundary conditions for both ordinary quasi-differential and elliptic partial differential operators is made precise in 13, Section 5.1, particularly Definition 5.1 and Theorem 5.1].

Conjecture 5.5. Consider a boundary value problem for a Lagrange symmetric ordinary quasi-differential expression of order $n \geq 2$ on an interval $(a, b) \subseteq \mathbb{R}$, as in Section 4.2. Then a complete Lagrangian $\mathrm{L} \subset \mathrm{S}$ (or a corresponding self-adjoint operator $T$ on $\left.D(T) \subset L^{2}((a, b) ; w)\right)$ is locally determined if and only if $\mathrm{L}$ is strictly separated, i.e.

$$
\text { Nec-coupling }(\mathrm{L})=0 \text {, }
$$

as in (4.15); otherwise $L$ is globally determined.

Example 5.6. In [13, Sections 3 and 4] the boundary value theory for certain elliptic partial differential expressions $A(x, D)$, defined in bounded regions $\Omega$ with smooth boundary $\partial \Omega$ in Euclidean space $\mathbb{E}^{r}(r \geq 2)$, is developed through symplectic algebra; compare Section 2.2. In particular the Dirichlet, Neumann, and 
Harmonic Lagrangians, and corresponding self-adjoint operators, are defined for such bounded regions $\Omega \subset \mathbb{E}^{r}$. The Dirichlet and Neumann boundary conditions specify locally determined self-adjoint operators (compare [13, Section 5.1, Theorem 5.1]). Furthermore, if a self-adjoint operator $T$ on $D(T)$ is defined via restrictions on elements of the maximal domain $D\left(T_{1}\right)$ by a system of boundary differential operators (see [13, Section 3, Definitions 3.1 and 3.2] involving functions and their normal derivatives evaluated on the boundary $\partial \Omega$ ), then $T$ is locally determined. On the other hand, it is known [13, Section 5, Theorem 5.1] that the Harmonic operator $T_{H a r}$ is globally determined, at least for the important special case of the classical Laplace operator $\Delta$ on a bounded region whose boundary $\partial \Omega$ is not connected.

Problem 5.7. Is $T_{\mathrm{Har}}$ globally determined when $\partial \Omega$ is connected?

The boundary $\partial \Omega$ of the bounded regions $\Omega \subset \mathbb{E}^{r}$, in our situation, consists of the (connected) components $\partial \Omega_{0}$ (the exterior hypersurface) and $\partial \Omega_{1}, \ldots, \partial \Omega_{k}$ (the interior hypersurfaces, if any). In the case when $\partial \Omega$ is not connected, say for the planar annular region [13, Section 4, Example 4.4] where $\partial \Omega_{0}$ and $\partial \Omega_{1}$ are concentric circles in $\mathbb{E}^{2}$, it is possible to specify differential boundary operators analogous to coupled boundary conditions, so defining a (essentially) self-adjoint operator. However, very little is known about such coupled boundary conditions for elliptic boundary value problems, but even allowing for the possibility of such coupled differential boundary operators, the Harmonic operator remains elusively unspecified by any kind of boundary evaluations.

Recent research (see [16) has uncovered a continuum of distinct analogues of the operator $T_{\text {Har }}$, that is self-adjoint extensions of the minimal operator generated by the Laplace differential expression $\Delta$ on the unit disk $\Omega \subset \mathbb{E}^{2}$ (see Example [5.6), each of which has a domain not entirely contained within the Sobolev space $W^{2}(\Omega)$. Hence these domains must contain some functions having no boundary values, in the sense of the trace map; see (2.42) and [13, Appendix A].

It remains an open problem to produce a coherent, and plausibly explicit, description of the set of all self-adjoint operators generated by the Laplace differential expression $\Delta$ on the unit disk $\Omega \subset \mathbb{E}^{2}$, although a good start may be the GKN-EM Theorem 1.14,

\section{REFERENCES}

[1] Abraham, R. and Marsden, J.E., Foundations of mechanics. (Benjamin/Cummings Publishing Co., Reading, MA: 1978.) MR0515141 (81e:58025)

[2] Akhiezer, N.I. and Glazman, I.M., Theory of linear operators in Hilbert space: I and II. (Pitman and Scottish Academic Press, London: 1981; translated from the third Russian edition of 1977.) MR0615736(83i:47001a), MR0615737 (83i:47001b)

[3] Bognár, J., Indefinite inner product spaces. (Springer-Verlag, Heidelberg: 1974.) MR0467261 $(57: 7125)$

[4] Birkhoff, G. and Mac Lane, S.A., A survey of modern algebra. (Macmillan Co., New York: 1953.) MR0054551(14,939a)

[5] Coddington, E.A. and Levinson, N., Theory of ordinary differential equations. (McGraw-Hill, New York: 1955.) MR0069338(16:1022b)

[6] Dunford, N. and Schwartz, J.T., Linear operators: I and II. (Wiley, New York: 1963.) MR0188745 (32:6181) 
[7] Everitt, W.N., On the deficiency index problem for ordinary differential operators: 19101977. Proceedings of the 1977 Uppsala International Conference on Differential Equations: 62-81; University of Uppsala, Sweden. (Distributed by Alquist and Wiksell International, Stockholm, Sweden: 1978.) MR0477247 (57:16788)

[8] Everitt, W.N., Linear ordinary quasi-differential expressions. Proceedings of the 1983 Beijing Symposium on Differential Equations and Differential Geometry: 1-28; University of Beijing. (Science Press, University of Beijing, P.R. China: 1986.) MR0880869

[9] Everitt, W.N. and Markus, L., The Glazman-Krein-Naimark theorem for ordinary differential operators. Operator Theory: Advances and Applications 98 (1997), 118-130. MR1478469 (99c:47070)

[10] Everitt, W.N. and Markus, L., Boundary value problems and symplectic algebra for ordinary differential and quasi-differential operators. Mathematical Surveys and Monographs, 61. (American Mathematical Society, Providence, RI: 1999.) MR.1647856 (2000c:34030)

[11] Everitt, W.N. and Markus, L., Complex symmetric geometry with applications to ordinary differential operators. Trans. Amer. Math. Soc. 351 (1999), 4905-4945. MR1637066 (2000c:34031)

[12] Everitt, W.N. and Markus, L., Multi-interval linear ordinary boundary value problems and complex symplectic algebra. Memoirs of the American Mathematical Society 151 (2001), Number 715. MR1828557 (2002i:34154)

[13] Everitt, W.N. and Markus, L., Elliptic partial differential operators and symplectic algebra. Memoirs of the American Mathematical Society 162 (2003), Number 770. MR.1955204 (2004d:47054)

[14] Everitt, W.N. and Markus, L., Infinite dimensional complex symplectic spaces. Memoirs of the American Mathematical Society 171 (2004), Number 810. MR2073219 (2005d:46048)

[15] Everitt, W.N., Markus, L. and Plum, M., An unusual self-adjoint linear partial differential operator. Trans. Amer. Math. Soc. 357 (2005), 1303-1324. MR2115367

[16] Everitt, W.N., Markus, L., Muzzulini, M. and Plum, M., A continuum of unusual self-adjoint elliptic partial differential operators. (In preparation.)

[17] Everitt, W.N. and Race, D., Some remarks on linear ordinary quasi-differential expressions. Proc. London Math. Soc. (3) 54 (1987), 300-320. MR0872809 (88b:34014)

[18] Everitt, W.N. and Zettl, A., Differential operators generated by a countable number of quasidifferential expressions on the real line. Proc. London Math. Soc. (3) 64 (1992), 524-544. MR.1152996 (93k:34182)

[19] Friedman, A., Partial differential equations. (Holt, Rinehart and Winston, New York: 1969.) MR0445088 (56:3433)

[20] Guillemin, V. and Sternberg, S., Symplectic techniques in physics. (Cambridge University Press: 1990.) MR.1066693 (91d:58073)

[21] Halmos, P. R., Naive set theory. (Van Nostrand Company, Inc., New York: 1960; reprinted Springer-Verlag, New York: 1974.) MR0453532 (56:11794)

[22] Kelley, J.L., General topology. (Van Nostrand Company, Inc., New York: 1955; reprinted Springer-Verlag, New York: 1975.) MR0370454 (51:6681)

[23] Markus, L., Hamiltonian dynamics and symplectic manifolds. (Lecture notes, University of Minnesota Bookstore (1973), 1-256.)

[24] Markus, L., Control of quasi-differential equations. Ann. Polon. Math. 51 (1990), 229-239. MR:1093993 (91m:93020)

[25] Markus, L., The Sturm-Liouville group. J. Comp. Appl. Math. 171 (2004), 335-365. MR2077212 (2005e:34071)

[26] McDuff, D. and Salamon, D., Introduction to symplectic topology. (Oxford University Press: 1995.) MR1373431 (97b:58062)

[27] Naimark, M.A., Linear differential operators: II. (Ungar, New York: 1968. Translated from the second Russian edition.) MR0262880(41:7485)

[28] Rudin, W. Functional analysis. (McGraw-Hill, New York: 1973; second edition: 1991.) MR.1157815 (92k:46001)

[29] Shin, D. On the solutions of a linear quasi-differential equation of order n. Mat. Sb. 7 (1940), 479-532. MR0003310 (2:198b)

[30] Smirnov, V.I., A course of higher mathematics, 5 (Pergamon Press Monographs in Pure and Applied Mathematics 62, Oxford: 1964.) MR0168707(29:5964) 
[31] Stone, M.H., Linear transformations in Hilbert space. American Mathematical Society Colloquium Publications, 15. (American Mathematical Society, Providence, Rhode Island: 1932.) MR 1451877 (99k:47001)

[32] Titchmarsh, E.C., Eigenfunction expansions, I. (Oxford University Press: second edition, 1962.) MR0176151 (31:426)

[33] Titchmarsh, E.C., Eigenfunction expansions, II. (Oxford University Press: 1958.) MR0094551 (20:1065)

[34] Wloka, J., Partial differential equations. (Cambridge University Press: 1987.) MR0895589 (88d:35004)

[35] Zettl, A., Formally self-adjoint quasi-differential operators. Rocky Mountain J. Math. 5 (1975), 453-474. MR0379976 (52:880)

School of Mathematics and Statistics, University of Birmingham, Edgbaston, BirmINGHAM B15 2TT, ENGLAND, UK

E-mail address: w.n.everitt@bham.ac.uk

School of Mathematics, University of Minnesota, Minneapolis, Minnesota 55455, USA

E-mail address: markus@math.umn.edu 